\title{
Modeling of wave overtopping on vertical structures with the HLLS Riemann solver
}

\author{
Matthew J. Wesley ${ }^{1}$, Kwok Fai Cheung ${ }^{*}$ \\ Department of Ocean and Resources Engineering, University of Hawaii at Manoa, Honolulu, \\ Hawaii 96822, USA
}

\begin{abstract}
Depth-integrated wave models are commonly used in coastal engineering design and studies of near-shore processes, but the results might be unstable or questionable when overtopping of vertical structures is involved. The HLLS Riemann solver presented by Murillo and GarciaNavarro (2012, Journal of Computational Physics) can account for shallow-water flow across a bottom step. We implement this solver in a second-order MUSCL-Hancock scheme and explore its use to describe overtopping of vertical structures through a nonlinear shallow-water model in one dimension. A series of numerical tests based on the Riemann problem assess the characteristic approximation of the flow resulting from two-dimensional processes at the step. The model gives good agreement with simulations from OpenFOAM (an open-source computational fluid dynamics code) for rarefaction and surge and qualitative description for reflection at the step and shock waves generated by free fall of water. A laboratory experiment was conducted in a $9.14 \mathrm{~m}$ long flume to provide validation data for solitary wave overtopping of a vertical structure. Consistent with the numerical tests, the model produces slightly larger reflected waves from the structure and underestimates the amplitude and speed of the downstream waves generated by overtopping. Despite its simplicity, the HLLS solver performs reasonably well in approximating the overall processes and produces stable and efficient numerical results for practical application.

\footnotetext{
* Corresponding author. Tel.: +1-808-956-3485; fax: +1-808-956-3498 E-mail: Matthew.Wesley@usace.army.mil (M.J. Wesley), cheung@ hawaii.edu (K.F. Cheung) ${ }^{1}$ Present address: US Army Corps of Engineers, Coastal Design Section, Los Angeles District, 915 Wilshire Blvd, Ste 930, Los Angeles, California 90017, USA
} 
Keywords: Bottom discontinuities, Finite Volume, Riemann solver, Shock-capturing, Vertical breakwater, Wave overtopping

\section{Introduction}

The capability to model overtopping of coastal structures by tsunamis, storm surge, and waves is important for engineering design and flood hazard assessment. Vertical breakwaters and seawalls are particularly challenging to numerical models. Computational fluid dynamics (CFD) codes can simulate overtopping of these structures with high fidelity. The computation, however, takes significant resources because of flow complexities involving vortex formation and airwater interactions and is typically utilized in local applications for load and scour prediction (e.g., Bricker et al., 2013; Yim et al., 2014). Depth-integrated shock-capturing models based on Boussinesq-type or non-hydrostatic formulations can describe coastal wave processes and flood hazards on a regional scale (e.g., Autuono et al., 2009; Roeber and Cheung, 2012; Shi et al., 2012; Yamazaki et al., 2009, 2011; Zijlema et al., 2011). Shocks come naturally with the solution to the Riemann problem formulated from conservation laws. Modeling of hydraulic jumps and breaking waves as shocks conserves volume and momentum, while accounting for energy dissipation without the use of predefined empirical coefficients. Recent advances, which enable approximation of discontinuous flows over a bottom step, have the potential to extend the depth-integrated approach for modeling of overtopping on vertical structures.

Shock-capturing schemes have a long record of application for modeling of discontinuous flows with depth-integrated equations. Godunov (1959) was the first to propose discretization of a conservative system by a series of control volumes and solving a Riemann problem at each interface to capture flow discontinuities. The exact solution to the Riemann problem typically involves a computationally-intensive, iterative scheme. Roe (1981) provided a local linearization that leads to an efficient and accurate approximation of the solution. Harten et al. (1983) proposed another commonly-used approximate solver, known as HLL (Harten, Lax, van Leer), from the integral form of conservation laws. These earlier works focus on dynamics of ideal 
gases that follow Euler's equations. Glaister (1988) and Toro (1992) extended the shockcapturing scheme to the nonlinear shallow-water equations. Brocchini et al. (2001) provided an efficient solver for nearshore flows based on the weighted average flux method, while Wei et al. (2006) implemented the surface-gradient method of Zhou et al. (2001) for runup modeling. With approximate Riemann solvers, Dodd (1998) investigated free surface flow across a breakwater with gentle side slopes and $\mathrm{Hu}$ et al. (2000) computed the overtopping rate on vertical structures by implementing a steep bottom slope on the front wall and an open boundary condition for the outgoing flow across the crest.

The aforementioned models and studies with shock-capturing schemes cater to surface and momentum discontinuities. Zhou et al. (2002) extended a Godunov-type scheme to include effects of a submerged bottom step. The energy change across the step is approximated by an empirical method and included in the source term of the nonlinear shallow-water equations. George (2008) instead included effects of the step as a forcing or source term in the Riemann problem and derived an approximate solver for the augmented system, which ensures conservation of flow momentum over irregular topography. The resulting two-dimensional model reproduces field and laboratory measurements of dam-break flows over rugged mountain terrain (George, 2011). Bernetti et al. (2008) approximated the force on a bottom step as hydrostatic and provided an exact solution to the augmented Riemann problem. Although exact, this solution is not unique in the presence of a bottom step and must be determined using other external factors such as energy principles and physical constraints (Alcrudo and Benkhaldoun, 2001). Murillo and Garcia-Navarro $(2010,2012)$ provided an approximate solution to this Riemann problem with an hydrostatic source term associated with the bottom step. Implementation of the resulting solver, known as HLLS ( $\mathrm{S}$ for step), in one and two-dimensional nonlinear shallow-water models produces good agreement with analytical solutions and laboratory measurements.

Application of bottom-discontinuous Riemann solvers has so far been limited to stepwise approximation of irregular topography for better conservation of the hyperbolic flow character. 
Although these solvers do not include vertical flows to physically describe overtopping of coastal structures, they can better approximate the resulting characteristics for coastal engineering design and flood hazard assessment. We implement the HLLS solver of Murillo and Garcia-Navarro (2012) in a nonlinear shallow-water model and examine its capability to account for free-surface flows across a vertical structure in one dimension. Their well-balanced scheme provides an accurate description of wetting and drying fronts as well as a steady-state preservation for a systematic examination of the solver. A two-fold approach allows assessment

of the resulting model in reproducing the characteristic flow patterns and in coastal engineering applications. The model is first applied to a series of numerical tests based on the Riemann problem with a bottom step and the results are compared with those from the open-source CFD code OpenFOAM (Weller et al., 1998). A laboratory flume experiment involving solitary wave overtopping of a vertical structure is conducted to assess the validity of the numerical approach in a practical setting.

\section{Mathematical Formulation}

With reference to Figure 1, a Cartesian coordinate system $(x, z)$ defines a free-surface flow varying with time $t$. Let $g$ denote acceleration due to gravity and $\rho$ the water density. The nonlinear shallow-water equations ensure conservation of mass and momentum in the flow, and when written in conservative form, read

$$
\mathbf{U}_{t}+\mathbf{F}(\mathbf{U})_{x}=\mathbf{S}
$$

in which $\mathbf{U}$ is the vector of conserved variables, $\mathbf{F}(\mathbf{U})$ is the flux vector, and $\mathbf{S}$ contains the source terms defined respectively as

$$
\begin{gathered}
\mathbf{U}=\left[\begin{array}{c}
h \\
h u
\end{array}\right] \\
\mathbf{F}(\mathbf{U})=\left[\begin{array}{c}
h u \\
h u^{2}+\frac{1}{2} g h^{2}
\end{array}\right] \\
\mathbf{S}=\left[\begin{array}{c}
0 \\
-g h \eta_{x}-\frac{\tau_{b}}{\rho}
\end{array}\right]
\end{gathered}
$$


where $u$ is horizontal velocity, $h$ is flow depth, $\eta$ is bottom elevation, and $\tau_{b}$ is bottom friction in terms of the Manning number. The free surface elevation is given by $\zeta=\eta+h$. The governing equation (1) in matrix form allows implementation of a Godunov-type scheme, which discretizes the domain into computational cells and utilizes a Riemann solver to impose conservation across flow discontinuities at each cell interface.

\subsection{Riemann Problem}

The Riemann problem consists of a hyperbolic system with two constant states separated by an initial discontinuity. The formulation typically excludes the bottom slope and friction, which are treated as source terms in a Godunov-type scheme. However, the slope becomes infinite at a step. If left untreated, unrealistic velocities would appear near the bottom discontinuity. Numerical models typically require smoothing of the topography to limit the slope within an acceptable range, but this is not always viable as in the case of a vertical structure. A more general approach is to reformulate the source term associated with the bottom slope for inclusion in the Riemann problem.

The definition sketch in Figure 2 places the initial flow discontinuity and the bottom step at $x$ $=0$ and denotes the left and right states by the subscripts $L$ and $R$. The step imposes a thrust on the incoming flow that is approximated by a hydrostatic pressure distribution as

$$
T=\rho g\left(h^{\prime}-\frac{\left|\Delta \eta^{\prime}\right|}{2}\right) \Delta \eta^{\prime}
$$

in which

$$
\begin{gathered}
h^{\prime}= \begin{cases}h_{L} & \text { if } \Delta \eta \geq 0 \\
h_{R} & \text { if } \Delta \eta<0\end{cases} \\
\Delta \eta^{\prime}=\left\{\begin{array}{lc}
h_{L} & \text { if } \Delta \eta \geq 0 \& \zeta_{L}<\eta_{R} \\
h_{R} & \text { if } \Delta \eta<0 \& \zeta_{R}<\eta_{L} \\
\Delta \eta & \text { otherwise }
\end{array}\right.
\end{gathered}
$$

where $\Delta \eta=\eta_{R^{-}} \eta_{L}$ is the step height (Bernetti et al., 2008). The hydrostatic pressure is literally determined by the flow depth facing the step. If the surface elevation on the step is higher, this 
approach underestimates the thrust on the flow. Murillo and Garcia-Navarro (2012) suggested to substitute the thrust term with $\rho g \tilde{h} \Delta \eta$ from George (2008), giving rise to

$$
T_{\text {max }}=\left\{\begin{array}{cc}
\min (\rho g \tilde{h} \Delta \eta, T) & \text { if } \Delta \zeta \Delta \eta \geq 0 \text { and } \tilde{u} \Delta \eta>0 \\
T & \text { otherwise }
\end{array}\right.
$$

where $\tilde{h}$ is the average water depth across the step, $\Delta \zeta=\zeta_{R}-\zeta_{L}$ denotes the initial surface discontinuity, and $\tilde{u}$ is the Roe (1981) averaged velocity of the left and right states.

The solution to the Riemann problem with the source term, proposed by Murillo and GarciaNavarro $(2010 ; 2012)$, begins with the weak form of the nonlinear shallow-water equations:

$$
\int_{0}^{\Delta t} \int_{x_{L}}^{x_{R}}\left[\mathbf{U}_{t}+\mathbf{F}(\mathbf{U})_{x}-\mathbf{S}\right] d x d t=\mathbf{0}
$$

where $\Delta t$ is a small time interval. Solving for the vector of conserved variables gives

$$
\int_{x_{L}}^{x_{R}} \mathbf{U}(x, \Delta t) d x=x_{R} \mathbf{U}_{R}-x_{L} \mathbf{U}_{L}-\left(\mathbf{F}_{R}-\mathbf{F}_{L}-\overline{\mathbf{S}}\right) \Delta t
$$

where $\overline{\mathbf{S}}=\left[0,-T_{\max } / \rho\right]^{\mathrm{T}}$ is the source term associated with the bottom step over the integrated area. The integral average of the vector of conserved variables is defined as

$$
\overline{\mathbf{U}}=\frac{\int_{x_{L}}^{x_{R}} \mathbf{U}(x, \Delta t) d x}{\Delta t\left(s_{R}-s_{L}\right)}
$$

which leads to the solution in the middle state or the star region

$$
\overline{\mathbf{U}}^{*}=\frac{s_{R} \mathbf{U}_{R}-s_{L} \mathbf{U}_{L}-\mathbf{F}_{R}+\mathbf{F}_{L}+\overline{\mathbf{S}}}{\left(s_{R}-s_{L}\right)}
$$

where $s$ is propagation speed. If $\overline{\mathbf{S}}=[0,0]^{\mathrm{T}}$, this corresponds to the HLL solver given by Toro (1992) for a flat bottom. Since the source term is not zero, the middle state has two distinct solutions on the left and right sides

$$
\begin{array}{r}
\mathbf{U}_{L}^{*}=\frac{\int_{x_{L}}^{0} \mathbf{U}(x, \Delta t) d x}{-\Delta t s_{L}} \\
\mathbf{U}_{R}^{*}=\frac{\int_{0}^{x_{R}} \mathbf{U}(x, \Delta t) d x}{\Delta t s_{R}}
\end{array}
$$

which are determined from the conserved quantities within the middle state. 
The energy across a shock wave must follow the Rankine-Hugoniot jump condition, which is also true for the two middle states and across the bottom step leading to

$$
\begin{gathered}
\mathbf{F}_{L}^{*}-\mathbf{F}_{L}=s_{L}\left(\mathbf{U}_{L}^{*}-\mathbf{U}_{L}\right) \\
\mathbf{F}_{R}-\mathbf{F}_{R}^{*}=s_{R}\left(\mathbf{U}_{R}-\mathbf{U}_{R}^{*}\right) \\
\mathbf{F}_{R}^{*}-\mathbf{F}_{L}^{*}=\overline{\mathbf{S}}
\end{gathered}
$$

If only shock waves are involved, the middle-state flux difference in equation (17) can be determined from linearization of the homogeneous governing equations as

$$
\mathbf{F}_{R}^{*}-\mathbf{F}_{L}^{*}=\mathbf{J}\left(\widetilde{\mathbf{U}}^{*}\right)\left(\mathbf{U}_{R}^{*}-\mathbf{U}_{L}^{*}\right)
$$

where $\widetilde{\mathbf{U}}$ is a Roe (1981) average and $\mathbf{J}$ denotes the Jacobian matrix. Since the star region is unknown a priori, the Jacobian is approximated with the initial state to give:

$$
\mathbf{J}(\widetilde{\mathbf{U}})\left(\mathbf{U}_{R}^{*}-\mathbf{U}_{L}^{*}\right)=\overline{\mathbf{S}}
$$

The simultaneous equations (15), (16), (17), and (19) give an explicit solution for the two middle states as

$$
\begin{gathered}
\mathbf{U}_{L}^{*}=\frac{s_{R} \mathbf{U}_{R}-s_{L} \mathbf{U}_{L}+\mathbf{F}_{L}-\mathbf{F}_{R}+\overline{\mathbf{S}}-s_{L} \overline{\mathbf{H}}}{s_{R}-s_{L}} \\
\mathbf{U}_{R}^{*}=\frac{s_{R} \mathbf{U}_{R}-s_{L} \mathbf{U}_{L}+\mathbf{F}_{L}-\mathbf{F}_{R}+\overline{\mathbf{S}}-s_{R} \overline{\mathbf{H}}}{s_{R}-s_{L}} \\
\mathbf{F}_{R}^{*}=\frac{s_{R} \mathbf{F}_{L}-s_{L} \mathbf{F}_{R}+s_{L} s_{R}\left(\mathbf{U}_{R}-\mathbf{U}_{L}\right)+s_{R}\left(\overline{\mathbf{S}}-s_{L} \overline{\mathbf{H}}\right)}{s_{R}-s_{L}} \\
\mathbf{F}_{L}^{*}=\frac{s_{R} \mathbf{F}_{L}-s_{L} \mathbf{F}_{R}+s_{L} s_{R}\left(\mathbf{U}_{R}-\mathbf{U}_{L}\right)+s_{L}\left(\overline{\mathbf{S}}-s_{R} \overline{\mathbf{H}}\right)}{s_{R}-s_{L}}
\end{gathered}
$$

where $\overline{\mathbf{H}}=\overline{\mathbf{S}} \mathbf{J}^{-1}$. Figure 3 illustrates the initial conditions and evolution of a typical solution to the Riemann problem with a bottom step. This solution consists of a stationary jump at the step and waves propagating away from the initial discontinuity in the form of a shock or rarefaction wave. A shock wave propagates at a characteristic speed according to the Rankine-Hugonoit 
conditions. A rarefaction wave provides a smooth transition from one state to another with a propagation speed given by the Riemann invariants.

\subsection{Godunov-type Scheme}

We utilize a Godunov-type scheme to solve the governing equation (1) for illustration and validation of the Riemann solver. Let $\Delta x$ and $\Delta t$ denote the cell and time step sizes. A secondorder upwind MUSCL-Hancock scheme integrates the conserved variables in time through a predictor and a corrector step as

$$
\begin{gathered}
\mathbf{U}_{i}^{n+\frac{1}{2}}=\mathbf{U}_{i}^{n}-\frac{\Delta t}{2 \Delta x}\left[\left(\left(\mathbf{F}_{L}\right)_{i+\frac{1}{2}}^{n}-\left(\mathbf{F}_{R}\right)_{i-\frac{1}{2}}^{n}\right)-\mathbf{S}_{i}^{n}\right] \\
\mathbf{U}_{i}^{n+1}=\mathbf{U}_{i}^{n}-\frac{\Delta t}{\Delta x}\left[\left(\left(\mathbf{F}^{-}\right)_{i+\frac{1}{2}}^{n+\frac{1}{2}}-\left(\mathbf{F}^{+}\right)_{i-\frac{1}{2}}^{n+\frac{1}{2}}\right)-\mathbf{S}_{i}^{n+\frac{1}{2}}\right]
\end{gathered}
$$

where the indices $i$ and $i \pm 1 / 2$ denote cell center and interface variables and $n$ is the current time step. With the topography incorporated into the Riemann problem, the source term $\mathbf{S}=$ $\left[0,-\tau_{b} / \rho\right]^{\mathrm{T}}$ includes the bottom friction only. The predictor step (24) evolves the conserved variables over a half time step based on the flux and source terms at the current time. The predicted variables revise the source term and along with the Riemann solver define the fluxes on the left and right sides of the interface, denoted by $\mathbf{F}^{-}$and $\mathbf{F}^{+}$in equation (25), for update of the flow over a full time step.

The HLLS solver proposed by Murillo and Garcia-Navarro (2012) can uniquely define the middle-state fluxes on the two sides of the step by equations (22) and (23). Since the flow cannot transition from sub- to super-critical within a cell or vice versa, the interface fluxes on the two sides are:

$$
\mathbf{F}_{i+\frac{1}{2}}^{-}=\left\{\begin{array}{cc}
\mathbf{F}_{L} & \text { if } 0 \leq s_{L} \\
\frac{s_{R} \mathbf{F}_{L}-s_{L} \mathbf{F}_{R}+s_{L} s_{R}\left(\mathbf{U}_{R}-\mathbf{U}_{L}\right)+s_{L}\left(\overline{\mathbf{S}}_{i+\frac{1}{2}}-s_{R} \overline{\mathbf{H}}_{i+\frac{1}{2}}\right)}{s_{R}-s_{L}} & \text { if } s_{L} \leq 0 \leq s_{R} \\
\mathbf{F}_{R}-\overline{\mathbf{S}}_{i+\frac{1}{2}} & \text { if } 0 \geq s_{R}
\end{array}\right.
$$




$$
\mathbf{F}_{i+\frac{1}{2}}^{+}=\left\{\begin{array}{cl}
\mathbf{F}_{L}+\overline{\mathbf{S}}_{i+\frac{1}{2}} & \text { if } 0 \leq s_{L} \\
\frac{s_{R} \mathbf{F}_{L}-s_{L} \mathbf{F}_{R}+s_{L} s_{R}\left(\mathbf{U}_{R}-\mathbf{U}_{L}\right)+s_{R}\left(\overline{\mathbf{S}}_{i+\frac{1}{2}}-s_{L} \overline{\mathbf{H}}_{i+\frac{1}{2}}\right)}{s_{R}-s_{L}} & \text { if } s_{L} \leq 0 \leq s_{R} \\
\mathbf{F}_{R} & \text { if } 0 \geq s_{R}
\end{array}\right.
$$

The presence of a bottom step requires the use of the Roe-averaged propagation speeds

$$
\begin{aligned}
& s_{L}= \begin{cases}\min \left(u_{L}-\sqrt{g h_{L}}, u^{*}-\sqrt{\phi^{*}}, \tilde{u}-\sqrt{g \tilde{h}}\right) & \text { if } \overline{\mathbf{S}}=0 \\
\tilde{u}-\sqrt{g \tilde{h}} & \text { if } \overline{\mathbf{S}} \neq 0\end{cases} \\
& s_{R}= \begin{cases}\max \left(u_{R}+\sqrt{g h_{R}}, u^{*}+\sqrt{\phi^{*}}, \tilde{u}+\sqrt{g \tilde{h}}\right) & \text { if } \overline{\mathbf{S}}=0 \\
\tilde{u}+\sqrt{g \tilde{h}} & \text { if } \overline{\mathbf{S}} \neq 0\end{cases}
\end{aligned}
$$

in which

$$
\begin{gathered}
u^{*}=\frac{u_{L}+u_{R}}{2}+\sqrt{g h_{L}}-\sqrt{g h_{R}} \\
\sqrt{\phi^{*}}=\frac{\sqrt{g h_{L}}+\sqrt{g h_{R}}}{2}+\frac{u_{L}-u_{R}}{4}
\end{gathered}
$$

When a dry cell is to the right or left of a wet cell, the propagation speeds are modified to:

$$
\begin{gathered}
s_{L}=u_{L}-\sqrt{g h_{L}} \quad s_{R}=u_{L}+2 \sqrt{g h_{L}} \\
s_{L}=u_{R}-2 \sqrt{g h_{R}} \quad s_{R}=u_{R}+\sqrt{g h_{R}}
\end{gathered}
$$

according to $\mathrm{Hu}$ et al. (2000), except if adjacent to a step, where the Roe-averaged values must be used.

Both the predictor and corrector steps involve reconstruction of the interface fluxes from the conserved variables at the cell centers. The second-order scheme defines the variables as piecewise linear in each cell. The surface gradient method proposed by Zhou et al. (2001) reconstructs the interface surface elevation instead of the flow depth to eliminate depth-interpolation errors. To reduce spurious oscillations, a slope limiter is applied to remove unrealistic gradients across 
discontinuities during variable reconstruction. The minmod limiter, which works well with the present problem, is applied to both the surface elevation and momentum before the computation of the interface fluxes. A bottom step can represent the front or back wall of a vertical structure for modeling of overtopping. Because of the large elevation difference between the adjacent cells, a second-order limiter provides unrealistic reconstructed values at the interface. A firstorder limiter with upwind or downwind bias is applied within the cells adjacent to the wall. The use of flow information from the respective side avoids creating an artificial gradient across the step. This local treatment is important for approximation of flows across a large bottom discontinuity.

\section{Comparison with CFD Model}

The Riemann problem includes effects of a bottom step as a source term, which is literally a horizontal thrust imposed on the flow. The physical processes as well as the solution remain one dimensional. Because the source term depends on the conditions immediately on the left and right of the interface that evolve with time and space, the HLLS solver can only be assessed numerically through a Godunov-type scheme. Although Murillo and Garcia-Navarro (2012) have verified the solver with the exact solution from Bernetti et al. (2008), it is necessary to examine its validity in describing flows resulting from predominantly two-dimensional processes at the step. We examine the nonlinear shallow-water (NSW) model with a series of test cases, which represent basic features of the Riemann problem relevant to overtopping of a vertical structure. The results are compared with the solution found by OpenFOAM, which fully accounts for the vertical flow structure including air-water interactions.

\subsection{Model Setup}

OpenFOAM (Open source Field Operation and Manipulation) is an open-source CFD toolbox that enables customization of applications in continuum mechanics and chemical processes. The InterFOAM solver within OpenFOAM makes use of the volume of fluid (VoF) 
technique to track the interface of a two-phase flow (Weller et al., 1998). A scalar function defines the ratio of air to water in each computational cell and typically a value of 0.5 delineates the free surface. With sufficient resolution, this technique can resolve splashing and air entrainment to realistically describe the flow field across a large bottom step. This feature is important for validation of the HLLS solver through the NSW model. The InterFOAM module solves the Navier-Stokes equations for each phase simultaneously. For this application, the fluid viscosity is set to zero. The resulting Euler equations in two dimensions are utilized for consistency with the inviscid fluid assumption in the Riemann problem.

Table 1 lists the initial conditions of the test cases that comprise the left and right states separated by a discontinuity as illustrated in Figure 2. Each test case is devised to mimic or isolate an aspect of the Riemann problem for direct comparison of the NSW and OpenFOAM solutions. The computational domains correspond to a flume of $60 \mathrm{~m}$ long and $2.5 \mathrm{~m}$ high with the bottom step at the center. The height of the domain is not a factor in the one-dimensional NSW model and the vertical wall of the step is modeled by the interface of the two adjacent cells. The initial conditions also define the steady upstream boundary conditions on the left side, while a reflective condition is imposed at the downstream boundary. Bed friction is not considered in the NSW model, while the free slip condition and a zero pressure gradient are applied at all boundaries in OpenFOAM. Sensitivity tests showed a grid size of $0.05 \mathrm{~m}$ in the NSW model can accurately capture the shock waves and a grid size of $0.02 \mathrm{~m}$ by $0.02 \mathrm{~m}$ in OpenFOAM can provide converging numerical results for the test cases.

The computations were performed on an Intel I-7 processor for an elapsed time of 4 to $5 \mathrm{sec}$ before the characteristic flows reach the boundaries. The time step is determined from a Courant number of 0.5 for the NSW model and dynamically within OpenFOAM to maintain a stable solution. OpenFOAM was compiled and executed in the serial mode to compare with the computing time from the NSW model. OpenFOAM takes approximately $8 \mathrm{hr}$ for a $5 \mathrm{sec}$ simulation, while the NSW model can complete the calculation with less than 4 min in the Matlab environment. The computational cost of OpenFOAM will be substantially higher if a 
turbulence model is included. Although the computing time depends on many factors that vary between the two models and their operating environments, the results provide a general indication of the relative computational requirements. The NSW model provides a highly efficient solution to the overtopping problem if only the characteristic flow patterns are of interest.

\subsection{Results and Discussion}

We first examine the performance of the HLLS solver in two basic discontinuous flow problems with a flat bottom. Figure 4 plots the initial surface elevations and the NSW and OpenFOAM results at $t=5 \mathrm{~s}$. The standard dam-break problem in case 1 produces a rarefaction wave propagating to the left and a shock wave to the right. Although the NSW model cannot describe the transient oscillations and entrapped air as shown in Figure 4a, the computed propagation speeds and the middle-state water depth follow closely with the more complete solution from OpenFOAM. For a uniform surge against a vertical wall in case 2, overtopping should not occur and implementation of ghost cells behind the wall would produce a correct shallow-water solution. However, the source term does not take into account the inertia force from sudden deceleration of the incoming flow at the wall. As pointed out by Murillo and Garcia-Navarro (2012), the HLLS solver cannot balance the flux and produces some initial leakage of water up the step as depicted in Figure 4b. The comparison of the source term with the integrated pressure from OpenFOAM in Table 2 shows the pressure on the wall is nearly hydrostatic by $t=5 \mathrm{~s}$. The NSW model produces a reflected shock wave from the wall. The propagation speed and flow depth agree quite well with OpenFOAM in spite of the leakage across the step and the lack of undulation behind the wave front.

Cases 3 and 4 correspond to the standard dam break over a submerged step in opposing directions to illustrate the basic characteristics of the Riemann problem. Figure 5 shows the initial surface elevations, bottom profiles, and the model results at $t=5 \mathrm{~s}$. The NSW model produces a stationary jump at the step instantly from the initial conditions in both cases. 
OpenFOAM shows gradual development of the middle state and produces a near-stationary wave in lieu of a jump slightly downstream of the step in $5 \mathrm{~s}$. The two models give good agreement of the heights and speeds of the rarefaction and shock. However, the OpenFOAM results show modulation of the rarefaction wave propagating toward the left in Figure 5a and the shock wave toward the right in $5 \mathrm{~b}$ due to the transient, two-dimensional flows developed on the respective sides of the step. Since the hydrostatic assumption in the source term was originally made for the step up against the flow, the middle state from case 3 closely follows the solution from OpenFOAM. The source term gives excellent agreement with the integrated pressure on the step as indicated in Table 2. For case 4 with the step down, the hydrostatic assumption slightly underestimates the source term. The HLLS solver handles the overall flows reasonably well despite the transient circulations developed by the step in both cases.

Figure 6 shows the results for Cases 5 and 6, which involve flows across a step onto an initially dry bed. Case 5 has an initial still-water condition. Both models produce a jump at the step and a rarefaction wave propagating toward the left as shown in Figure 6a. OpenFOAM shows gradual development of the jump and noticeable modulation of the rarefaction from the transient circulation by the step. With an initial velocity in case 6 , the NSW model produces a stationary jump at the step as well as a reflected shock wave instantly. The middle state and shock height remain steady throughout the simulation. The sequence of snapshots in Figure $6 \mathrm{~b}$ indicates the jump and reflection from OpenFOAM develop gradually from the incoming flow redirected upward by the step. The jump increases over time reaching the same level as the NSW prediction. The reflected wave subsequently develops to a steady state with a greater height than the shock from the NSW model. The hydrostatic assumption deviates when a circulation develops in front of the step. As indicated in Table 2, the source term underestimates the force on the step especially for case 6 with an initial velocity and air entrapment. Despite the discrepancies in the upstream flow, the NSW model gives excellent agreement with OpenFOAM for the surge on the dry bed in both cases. 
Figures $7 \mathrm{a}$ and $7 \mathrm{~b}$ show the results for cases 7 and 8 , which involve free fall of water without and with initial velocity from a step onto calm water. The NSW model gives an accurate account of the rarefaction waves and thus the overtopping rates in both cases, but underestimates the propagation speed of the shock on the downstream side despite a reasonable prediction of its height. The waterfall generates a supercritical flow and a hydraulic jump with extensive air-water interactions that likely contribute to the higher shock speed as seen in the OpenFOAM prediction. When the downstream side is initially dry as in cases 9 and 10, the OpenFOAM results in Figures $8 \mathrm{a}$ and $8 \mathrm{~b}$ show detachment of the waterfall from the wall. The impinging jet produces an unsteady air-water circulation by the step and a surge in the downstream direction. The NSW model produces a steady flow from the step that gradually transitions into a surge. The source term in these cases does not correspond to the forces acting on the step as seen in Table 2, but rather provides a mechanism to account for the potential energy of the waterfall and its contribution to the momentum of the downstream flow. Although the formulation of the Riemann problem does not account for the waterfall, the NSW model gives good qualitative agreement with OpenFOAM for the resulting surge.

\section{Comparison with Laboratory Model}

The HLLS solver provides a reasonable description of the characteristic flows generated from the bottom step. Laboratory experiments can complement the validation by assessing its performance in more realistic settings. George (2011) and Murillo and Garcia-Navarro (2012), who compared their model results with laboratory data of dam-break flows and wave runup, did not fully explore their solvers for modeling of overtopping. Although there are prior laboratory experiments related to overtopping of vertical structures (Franco and Franco, 1999; Pullen et al., 2009), the published datasets do not include measurements of the reflected and transmitted waves in a form suitable for model validation. Therefore, we conducted a laboratory experiment in the Hydraulics Laboratory of the Department of Civil and Environmental Engineering, University of Hawaii to further investigate the validity of the solver in practical application. 


\subsection{Laboratory and Numerical Model Setup}

The laboratory experiment provided measurements of the reflection and transmission as well as the mixing processes from overtopping of a vertical structure by solitary waves. Figure 9 illustrates the setup of the experiment and instrumentation. The flume is $9.14 \mathrm{~m} \mathrm{long}, 0.1524 \mathrm{~m}$ wide, and $0.39 \mathrm{~m}$ high with clear acrylic walls. The vertical structure, which represents a breakwater or levee with a rectangular cross section, is $0.0762 \mathrm{~m}$ wide and $0.1524 \mathrm{~m}$ tall made from clear acrylic. A piston-type wavemaker generates the incident solitary wave, which allows precise measurements of the flow characteristics for model validation (e.g., Hsiao et al., 2008; Roeber and Cheung, 2012; Quiroga and Cheung, 2013). The incident wave height $a$ is measured from the still-water level. Three capacitance-type wave gauges manufactured by JFE Advantech Co., Ltd. sample the surface elevations at $76 \mathrm{~Hz}$ with an uncertainty of $5 \times 10^{-5} \mathrm{~m}$. Gauge 1 in front of the structure provides measurements of the incident and reflected waves. Gauge 2 is placed just outside the downstream mixing zone to capture the regenerated waves, while gauge 3 records the wave characteristics downstream. The wave gauges are connected to a data acquisition system controlled by the WinLabEM software.

The clear acrylic flume allows illumination of the air-water interface with LED lights placed underneath and provides unobstructed views of the flow during the experiment. A high-speed camera with a Nikkor 50mm f/1.8D lens manufactured by Canadian Photonic Labs Inc. captures the overtopping and mixing processes at $400 \mathrm{fps}$. The camera is placed at $2 \mathrm{~m}$ from the wave flume, at an elevation of $0.12192 \mathrm{~m}$ above the bottom, and a distance of $0.22 \mathrm{~m}$ downstream from the front wall of the vertical structure. The camera recorded the two-dimensional water surface across the flume because of the angle of the lens. We utilized the MATLAB Image Processing Toolbox to refine the image contrast and extract the surface profile on the front face of the flume for comparison with the model results. The post-processing of the high-speed video data includes correction for lens distortion and parallax error as well as mapping of pixel coordinates to real-world coordinates (Brady et al., 2004). The coordinate transformation may 
involve an error of up to $0.026 \mathrm{~m}$. The error is greatest near the edges of view and is much smaller at the image directly in front of the camera. The post-processed images, which have been cropped accordingly, allow examination of the mixing processes and their effects on wave regeneration in comparison with the NSW model results.

The NSW model covers a flume of $10 \mathrm{~m}$ long with the vertical structure located at the center. The one-dimensional model accounts for the front and back walls of the structure as opposing steps connected by computational cells along the crest. The incident solitary wave is defined as part of the initial conditions. Because a solitary wave has an infinite wavelength, it is necessary to truncate its tails for the finite computational domain. The initial wave is positioned along the flume such that the surface elevation at the structure is less than $1 \%$ of the wave height. Numerical dispersion, which depends on the spatial discretization, is needed to maintain the incident solitary wave profile in the NSW model. The grid size on the upstream side requires a large value to provide sufficient dispersion for the given water depth (Yoon et al., 2007), but is limited to $4.5 \mathrm{~cm}$ for resolution of the wave processes. The structure and the downstream side have a much finer grid of $9.5 \mathrm{~mm}$ to resolve the more complex flow patterns. A Manning number of $0.009 \mathrm{~s} / \mathrm{m}^{1 / 3}$ is used to account for the friction on the acrylic surface. An open boundary condition is imposed at both ends of the computational domain.

\subsection{Results and Discussion}

A total of 30 tests were conducted with a combination of water depths on the two sides of the vertical structure and a range of incident solitary wave heights to examine the reflection, overtopping, and downstream processes. Because of the small-scale experiment, only tests with large relative wave heights $a / h_{1}$ produce measurements sufficiently outside the range of instrument uncertainties for model validation. Table 3 lists five selected test cases for illustration of the physical processes and assessment of the model. Tests 1 to 3 examine the effects of the downstream water depth on wave regeneration and propagation after overtopping. Tests 4 and 5 are variations of test 2 to investigate effects of reflection and nonlinearity. As expected in actual 
occurrences, four of the five selected tests involve extensive air entrainment, splashing, and turbulence from overtopping of the structure. These realistic conditions allow evaluation of the model performance in practical application.

Test 1 has the same water depth on both sides equal to the height of the structure and the incident wave condition of $a / h_{1}=0.3$. The overtopping does not produce a waterfall nor large air bubbles in the downstream flow. Figure 10 compares the computed and recorded surface elevations. At gauge 1 in front of the structure, the NSW model provides a good depiction of the incident wave profile. The larger and more abrupt reflection from the front wall, as indicated by the second peak, results from the HLLS solver, in which the shock approximation overestimates the reflected wave height under transient conditions (see case 6 in Section 3). The incident wave subsequently transforms into a surge on the structure that in turn transitions from supercritical to subcritical across the back wall. The video image shows good overall agreement of the model results with the measurements except near the structure, where the actual transition is more gradual and further downstream due to formation of a submerged jet above initially stagnant water. Gauges 2 recorded a transient initial pulse generated by the jet and surface disturbances associated with unsteady circulations in the mixing zone. The measurements at gauge 3 show a well-developed solitary wave followed by trailing oscillations with diminishing amplitude. The NSW model produces a solitary wave of comparable amplitude and phase but with steepened front face due to the lack of dispersion.

Test 2 has the same initial condition as test 1 in front of the structure, but half of the water depth downstream to produce a waterfall after overtopping. Figure 11 shows the results at gauge 1 are not sensitive to the downstream flow and are close to those in test 1 . Gauge 2 recorded a large leading wave followed by surface disturbances generated by the mixing processes, while the measurements at gauge 3 show two distinct solitary waves. The video image shows a jet from the crest of the structure impinging the water surface with air entrainment. The jet generates surface waves in both directions and reflection of the smaller upstream wave from the structure corroborates the formation of the second solitary wave observed at gauge 3 . The model gives a 


\section{Conclusions and Future Work}

The HLLS solver, which incorporates effects of a bottom step as a source term in the Riemann problem, has proved both efficacious and efficient in modeling of free surface flow over a vertical structure. Implementation of the solver in a one-dimensional, nonlinear shallowwater model allows representation of the front and back walls of the structure as opposing steps and incorporates their effects on the flow momentum as hydrostatic forces through the source term. This rather simple approach can greatly enhance the use of depth-integrated models for flood hazard mapping and coastal engineering design. However, precautions are deemed necessary in the interpretation of the model results due to the hydrostatic approximation of the source term, the shock assumption of the middle state, and the depth-integrated flow structure in the solver. 
A series of test cases mimicking the Riemann problem assesses the validity of the solver in reproducing the characteristic flow patterns across a step. Comparisons with OpenFOAM results show very good agreement for rarefaction waves developed upstream of the step and the downstream surge on initially dry bed. For an impulsive flow against a step, the hydrostatic force in the source term cannot fully balance the change in flow momentum resulting in leakage of water up the step. The middle-state shock assumption in the solver overestimates the initial height of partial reflection from the step, where the flow is actually redirected upward in a more gradual process. Free fall of the flow onto calm water involves extensive air entrainment and splashing in addition to rapid transitions of flow regimes by the step. The absence of these transient processes in the model results in a steady shock downstream and underestimation of its propagation speed.

A specially-designed laboratory experiment allows evaluation of the HLLS solver for practical application in a realistic setting. The solver produces stable numerical results for overtopping of a solitary wave on a vertical structure that can be challenging for conventional depth-integrated models. Despite the simplicity of the formulation, the model provides a good qualitative description of the primary physical processes, which include partial wave reflection from the front wall, surge formation on the crest, and downstream wave regeneration and propagation. The approach, however, overestimates the reflected wave height from the structure and underestimates the downstream propagation speed due to depth integration. The overall results improve with decreasing solitary wave height and increasing wavelength, supporting the use of the solver to model storm surge and tsunamis overtopping of vertical structures.

George (2011) and Murillo and Garcia-Navarro (2012) have implemented bottomdiscontinuous Riemann solvers to describe two-dimensional shallow-water flows over topographic features. The latter study shows implementation of the HLLS solver in a twodimensional model alleviates the limitation of the hydrostatic source term and reduces leakage of the flow over the step. Similar augmentation can be made to a non-hydrostatic or Boussinesq model to include effects of wave dispersion. Additional CFD and laboratory tests are necessary 
to evaluate the adequacy of the solver to describe overtopping of three-dimensional vertical structures. A large-scale laboratory experiment will better reproduce air-water interactions and mixing processes thereby minimizing scale effects to better capture the characteristic flow patterns for model validation.

\section{Acknowledgements}

The study was funded by the Office of Naval Research under Grant No. N00014-12-1-0721. Additional support was provided by the National Tsunami Hazard Mitigation Program under Grant No. NA09NWS4670016 through Hawaii Emergency Management Agency. We would like to thank the Department of Civil and Environmental Engineering, University of Hawaii for the use of the hydraulic laboratory and the two anonymous reviewers for their constructive criticisms that helped improve the presentation and highlight the contributions of this paper. SOEST Contribution No. XXXX.

\section{References}

Alcrudo, F., Benkhaldoun, F., 2001. Exact solution to the Riemann problem of the shallow water equations with discontinuous bottom geometry. Computers and Fluids 30(6), 634-671.

Antuono, M., Liapidevskii, V., Brocchini, M., 2009. Dispersive nonlinear shallow-water equations. Studies in Applied Mathematics 122(1), 1-28.

Bernetti, R., Titarev, V.A., Toro, E.F., 2008. Exact solution of the Riemann problem for the shallow water equations with discontinuous bottom geometry. Journal of Computational Physics 227(6), 3212-3243.

Brady, P.D.M., Boutounet, M., Beecham, S., 2004. Free surface monitoring using image processing. Proceedings of $15^{\text {th }}$ Australasian Fluid Mechanics Conference, University of Sydney, Sydney, Australia.

Bricker, J.D., Takagi, H., Mitsui, J., 2013. Turbulence model effects on VOF analysis of breakwater overtopping during the 2011 great east Japan tsunami. IAHR World Congress, Chengdu, China.

Brocchini, M., Bernetti, R., Mancinelli, A., and Albertini, G., 2001. An efficient solver for nearshore flows based on the WAF method. Coastal Engineering 43(2), 105-129.

Dodd, N., 1998. Numerical model of wave run-up, overtopping, and regeneration. Journal of Waterway, Port, Coastal, and Ocean Engineering 124(2), 73-81.

Franco, C., Franco, L., 1999. Overtopping formulas for caisson breakwaters with nonbreaking 3D waves. Journal of Waterway, Port, Coastal, and Ocean Engineering 125(2), 98-108. 
George, D.L., 2008. Augmented Riemann solvers for the shallow water equations over variable topography with steady states and inundation. Journal of Computational Physics 227(6), 3089-3113.

George, D.L., 2011. Adaptive finite volume methods with well-balanced Riemann solvers for modeling floods in rugged terrain: Application to the Malpasset dam-break flood. International journal for Numerical Methods in Fluids 66(8), 1000-1018.

Glaister, P., 1988. Approximate Riemann solutions of the shallow water equations. Journal of Hydraulic Research 26(3), 293-306.

Godunov, S.K., 1959. A difference method for numerical calculation of discontinuous solutions of the equations of hydrodynamics. Matematicheskii Sbornik 89(3), 271-306.

Harten, A., Lax, P.D., van Leer, B., 1983. On upstream differencing and Godunov-type schemes for hyperbolic conservation laws. SIAM Review 25(1), 35-61.

Hsiao, S.-C., Hsu, T.-W., Lin, T.-C., Chang, Y.-H., 2008. On the evolution and run-up of breaking solitary waves on a mild sloping beach. Coastal Engineering 55(12), 975-988.

Hu, K., Mingham, C.G., Causon, D.M., 2000. Numerical simulations of wave overtopping of coastal structures using the non-linear shallow water equations. Coastal Engineering 41(4), 433-465.

Murillo, J., Garcia-Navarro, P., 2010. Weak solutions for partial differential equations with source terms: Application to the shallow water equations. Journal of Computational Physics 229(11), 4327-4368.

Murillo, J., Garcia-Navarro, P., 2012. Augmented versions of the HLL and HLLC Riemann solvers including source terms in one and two dimensions for shallow flow applications. Journal of Computational Physics 231(20), 6861-6906.

Pullen, T., Allsop, W., Bruce, T., Pearson, J., 2009. Field and laboratory measurements of mean overtopping discharges and spatial distributions at vertical seawalls. Coastal Engineering 56(2), 121-140.

Quiroga, P.D., Cheung, K.F., 2013. Laboratory study of solitary-wave transformation over bedform roughness on fringing reefs. Coastal Engineering 80, 35-48.

Roe, P.L., 1981. Approximate Riemann solver, parameter vectors, and difference schemes. Journal of Computational Physics 43(2), 357-372.

Roeber, V., Cheung, K.F., 2012. Boussinesq-type model for energetic breaking waves in fringing reef environment. Coastal Engineering 70, 1-20.

Shi, F., Kirby, J.T., Harris, J.C., Geiman, J.D., Grilli, S.T., 2012. A high-order adaptive timestepping TVD solver for Boussinesq modeling of breaking waves and coastal inundation. Ocean Modelling 43-44, 36-51.

Toro, E.F., 1992. Riemann problems and the WAF method for solving the two-dimensional shallow water equations. Philosophical Transactions of the Royal Society of London A: Physical Sciences and Engineering 338(1649), 43-68.

Weller, H.G., Tabor, G., Jasak, H., Fureby, C., 1998. A tonsorial approach to computational continuum mechanics using object oriented techniques. Computers in Physics 12(6), 620631.

Yamazaki, Y., Kowalik, Z., Cheung, K.F., 2009. Depth-integrated, non-hydrostatic model for wave breaking and run-up. International Journal for Numerical Methods in Fluids 61(5), 473497. 
Yamazaki, Y., Cheung, K.F., and Kowalik, Z., 2011. Depth-integrated, non-hydrostatic model with grid nesting for tsunami generation, propagation, and run-up. International Journal for Numerical Methods in Fluids 67(12), 2081-2107.

Yim, S.C., Olsen, M.J., Cheung, K.F., Azadbakht, M., 2014. Tsunami modeling, fluid load simulation, and validation using geospatial field data. Journal of Structural Engineering 140. A4014012.

Yoon, S.B., Lim, C.H., Choi, J., 2007. Dispersion-correction finite difference model for simulation of transoceanic tsunamis. Terrestrial, Atmospheric and Oceanic Sciences 18(1), 31-53.

Wei, Y., Mao, X.-Z., Cheung, K.F., 2006. Well-balanced finite-volume model for long-wave runup. Journal of Waterway, Port, Coastal, and Ocean Engineering 132(2), 114-124.

Zhou, J.G., Causon, D.M., Ingram, D.M., Mingham, C.G., 2001. The surface gradient method for the treatment of source terms in the shallow-water equations. Journal of Computational Physics 168(1), 1-25.

Zhou, J.G., Causon, D.M., Ingram, D.M., Mingham, C.G., 2002. Numerical solutions of the shallow water equations with discontinuous bed topography. International Journal for Numerical Methods in Fluids 38(8), 769-788.

Zijlema, M., Stelling, G., and Smit, P., 2011. SWASH: An operational public domain code for simulating wave fields and rapidly varied flows in coastal waters. Coastal Engineering 58(10), 992-1012. 
Table 1. Initial conditions of test cases against OpenFOAM

\begin{tabular}{|ccccccc|}
\hline Case & $\begin{array}{c}h_{L} \\
(\mathrm{~m})\end{array}$ & $\begin{array}{c}h_{R} \\
(\mathrm{~m})\end{array}$ & $\begin{array}{c}u_{L} \\
(\mathrm{~m} / \mathrm{s})\end{array}$ & $\begin{array}{c}u_{R} \\
(\mathrm{~m} / \mathrm{s})\end{array}$ & $\begin{array}{c}\eta_{L} \\
(\mathrm{~m})\end{array}$ & $\begin{array}{c}\eta_{R} \\
(\mathrm{~m})\end{array}$ \\
\hline 1 & 2 & 1 & 0 & 0 & 0 & 0 \\
2 & 0.5 & 0 & 0.5 & 0 & 0 & 1 \\
3 & 2 & 1.5 & 0 & 0 & 0 & 1 \\
4 & 2 & 1.5 & 0 & 0 & 1 & 0 \\
5 & 2 & 0 & 0 & 0 & 0 & 1 \\
6 & 2 & 0 & 2 & 0 & 0 & 1 \\
7 & 1 & 0.5 & 0 & 0 & 1 & 0 \\
8 & 1 & 0.5 & 1 & 0 & 1 & 0 \\
9 & 1 & 0 & 0 & 0 & 1 & 0 \\
10 & 1 & 0 & 1 & 0 & 1 & 0 \\
\hline
\end{tabular}


Table 2. Thrust exerted by the bottom step on the flow at $t=5 \mathrm{~s}$

\begin{tabular}{|cccc|}
\hline Case & $\begin{array}{c}\text { Step } \\
\text { Direction }\end{array}$ & $\begin{array}{c}T_{\text {OpenFOAM }} \\
(\mathrm{kN} / \mathrm{m})\end{array}$ & $\begin{array}{c}T_{\max } \\
(\mathrm{kN} / \mathrm{m})\end{array}$ \\
\hline 1 & N/A & $\mathrm{N} / \mathrm{A}$ & $\mathrm{N} / \mathrm{A}$ \\
2 & upstream & -1.87 & -1.86 \\
3 & upstream & -12.85 & -12.85 \\
4 & downstream & 11.29 & 10.68 \\
5 & upstream & -12.30 & -12.12 \\
6 & upstream & -19.14 & -17.74 \\
7 & downstream & 1.96 & 2.82 \\
8 & downstream & 3.19 & 3.57 \\
9 & downstream & 2.10 & 0.71 \\
10 & downstream & 2.80 & 1.20 \\
\hline
\end{tabular}


Table 3. Initial conditions for select laboratory tests

\begin{tabular}{|ccccc|}
\hline Test & $\begin{array}{c}h_{1} \\
(\mathrm{~m})\end{array}$ & $\begin{array}{c}h_{2} \\
(\mathrm{~m})\end{array}$ & $\begin{array}{c}a \\
(\mathrm{~m})\end{array}$ & $\mathrm{a} / h_{1}$ \\
\hline 1 & 0.1524 & 0.1524 & 0.0457 & 0.3 \\
2 & 0.1524 & 0.0762 & 0.0457 & 0.3 \\
3 & 0.1524 & 0.0000 & 0.0457 & 0.3 \\
4 & 0.1219 & 0.0610 & 0.0366 & 0.3 \\
5 & 0.1524 & 0.0762 & 0.0305 & 0.2 \\
\hline
\end{tabular}




\section{List of Figure Captions}

1. Definition sketch for shallow-water flow.

2. Definition sketch for the Riemann problem with a bottom step.

3. Schematic (bottom) and characteristic (top) of the Riemann solver with a bottom step.

4. Comparison of NSW model and OpenFOAM results. (a) Case 1. (b) Case 2. The dotted, dash, and solid lines denote the initial surface elevation, NSW model, and OpenFOAM.

5. Comparison of NSW model and OpenFOAM results. (a) Case 3. (b) Case 4. The dotted, dash, and solid lines denote the initial surface elevation, NSW model, and OpenFOAM.

6. Comparison of NSW model and OpenFOAM results. (a) Case 5. (b) Case 6. The dotted, dash, and solid lines denote the initial surface elevation, NSW model, and OpenFOAM.

7. Comparison of NSW model and OpenFOAM results. (a) Case 7. (b) Case 8. The dotted, dash, and solid lines denote the initial surface elevation, NSW model, and OpenFOAM.

8. Comparison of NSW model and OpenFOAM results. (a) Case 9. (b) Case 10. The dotted, dash, and solid lines denote the initial surface elevation, NSW model, and OpenFOAM.

9. Setup of laboratory flume experiment.

10. Comparison of numerical and laboratory results for Test 1. (a) Computed (dash line) and recorded (solid line) surface elevation time series at wave gauges. (b) Recorded image and computed surface profile (white circles). The water surface in the recorded image is highlighted as needed for ease of comparison. The dotted line indicates the initial surface elevation.

11. Comparison of numerical and laboratory results for Test 2. (a) Computed (dash line) and recorded (solid line) surface elevation time series at wave gauges. (b) Recorded image and computed surface profile (white circles). The water surface in the recorded image is highlighted as needed for ease of comparison. The dotted line indicates the initial surface elevation. 
12. Comparison of numerical and laboratory results for Test 3. (a) Computed (dash line) and recorded (solid line) surface elevation time series at wave gauges. (b) Recorded image and computed surface profile (white circles). The water surface in the recorded image is highlighted as needed for ease of comparison. The dotted line indicates the initial surface elevation.

13. Comparison of numerical and laboratory results for Test 4. (a) Computed (dash line) and recorded (solid line) surface elevation time series at wave gauges. (b) Recorded image and computed surface profile (white circles). The water surface in the recorded image is highlighted as needed for ease of comparison. The dotted line indicates the initial surface elevation.

14. Comparison of numerical and laboratory results for Test 5. (a) Computed (dash line) and recorded (solid line) surface elevation time series at wave gauges. (b) Recorded image and computed surface profile (white circles). The water surface in the recorded image is highlighted as needed for ease of comparison. The dotted line indicates the initial surface elevation. 


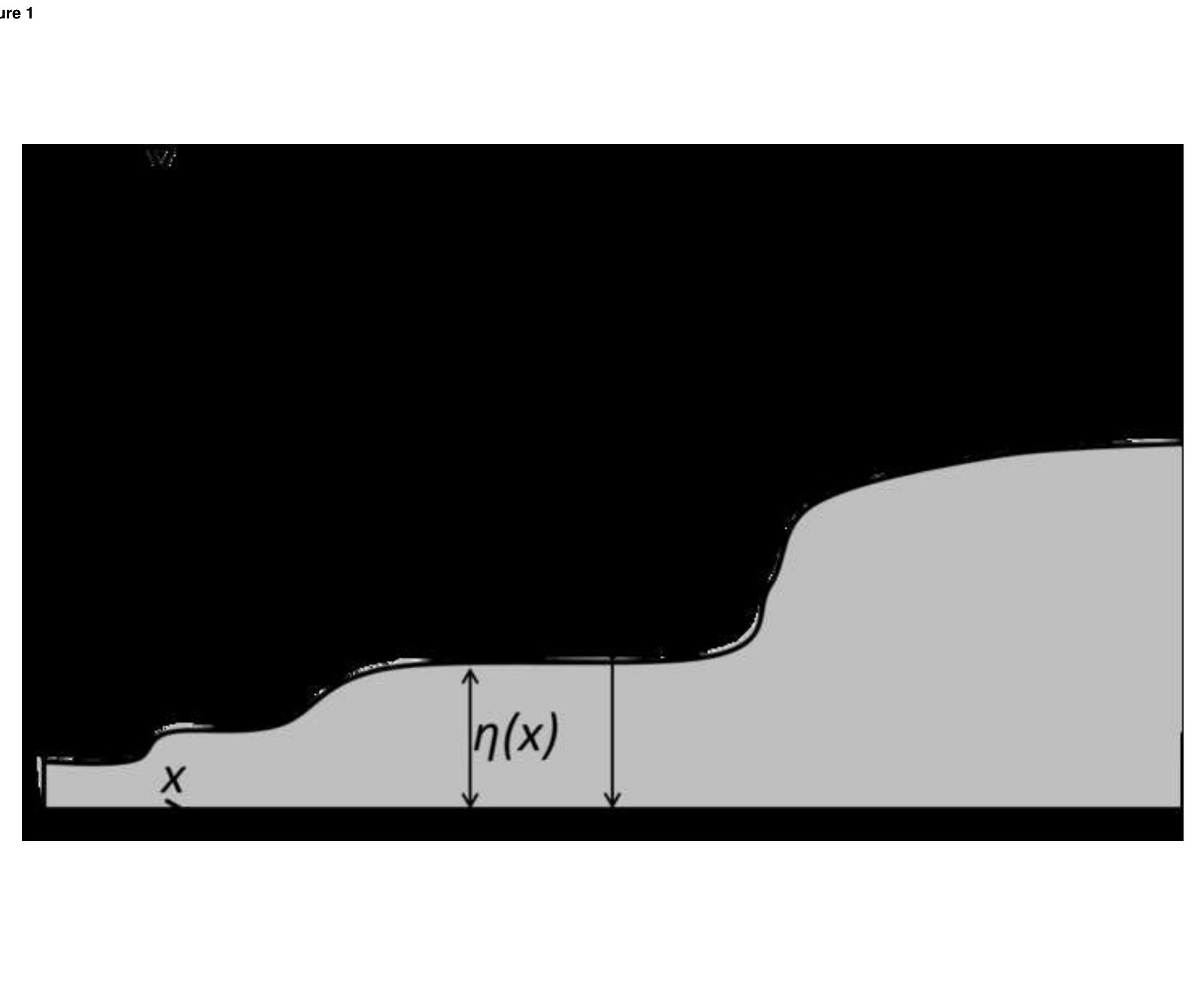

Finure

1

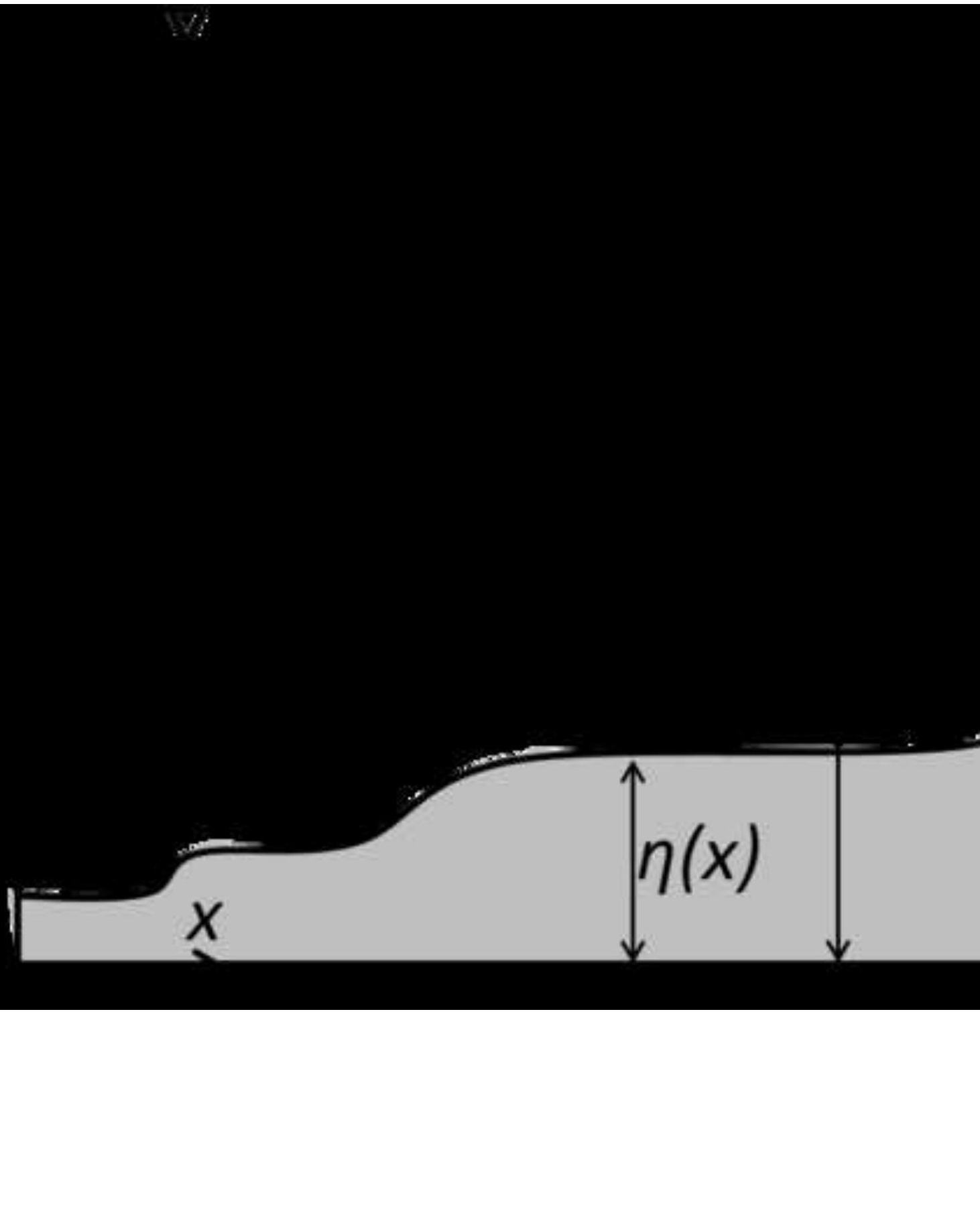

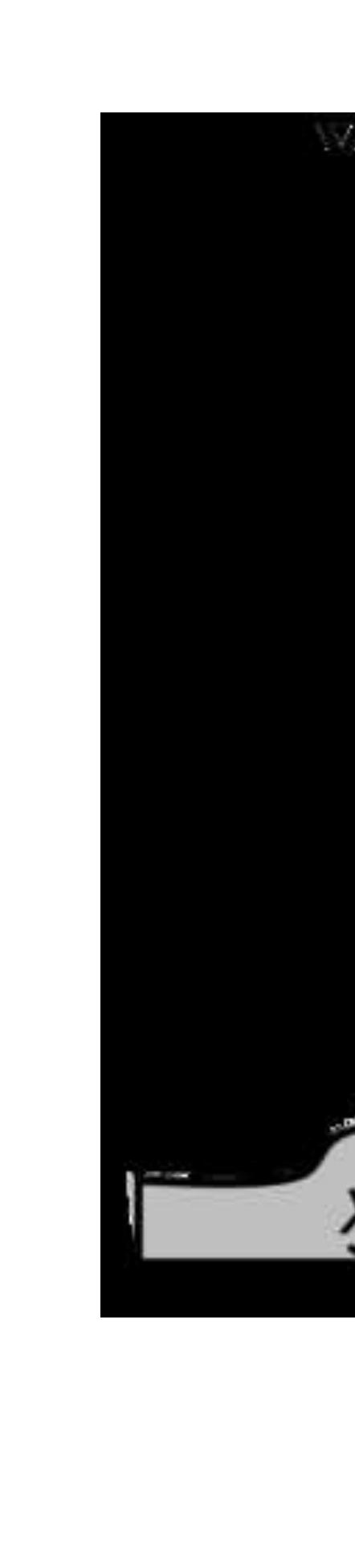
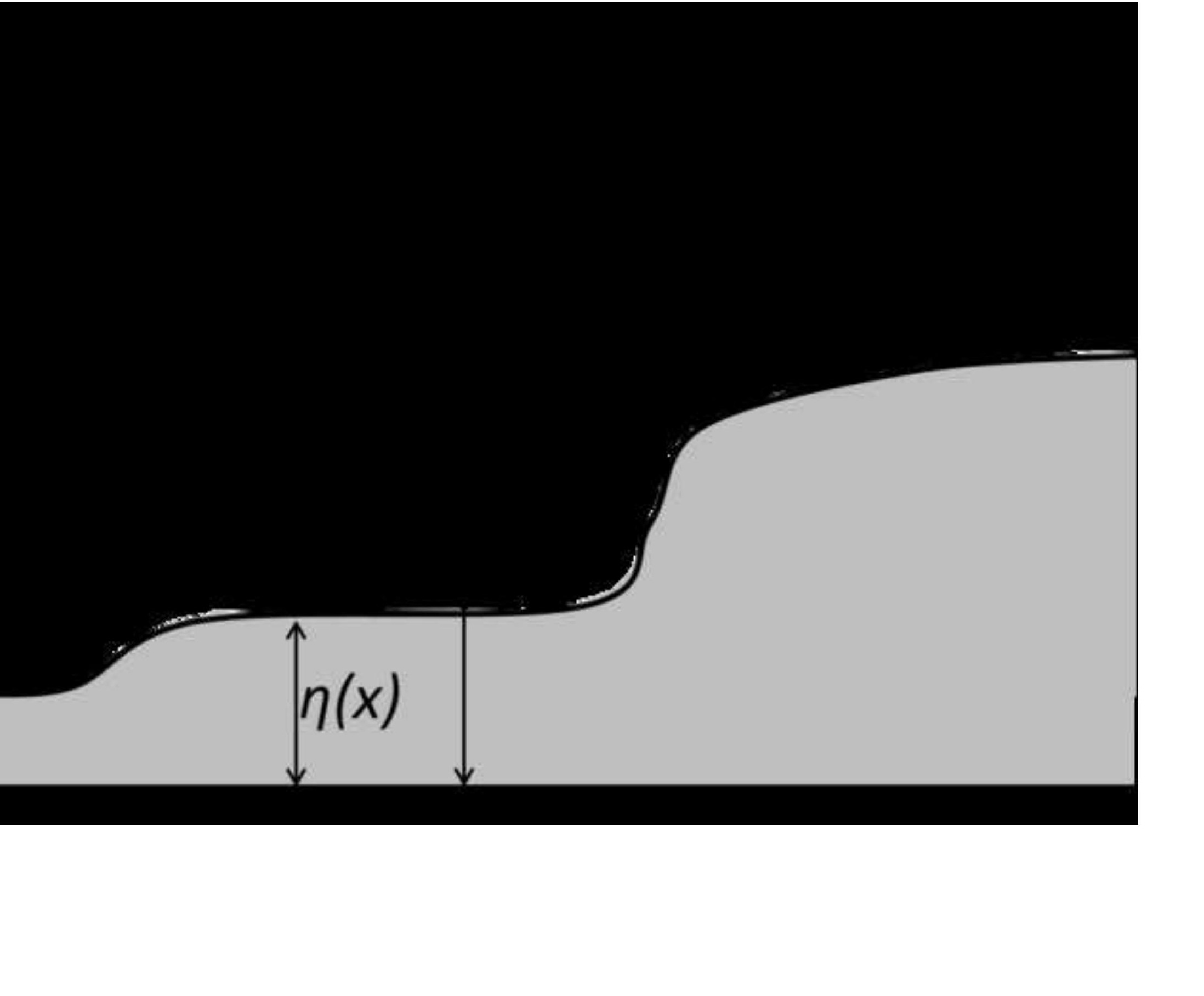

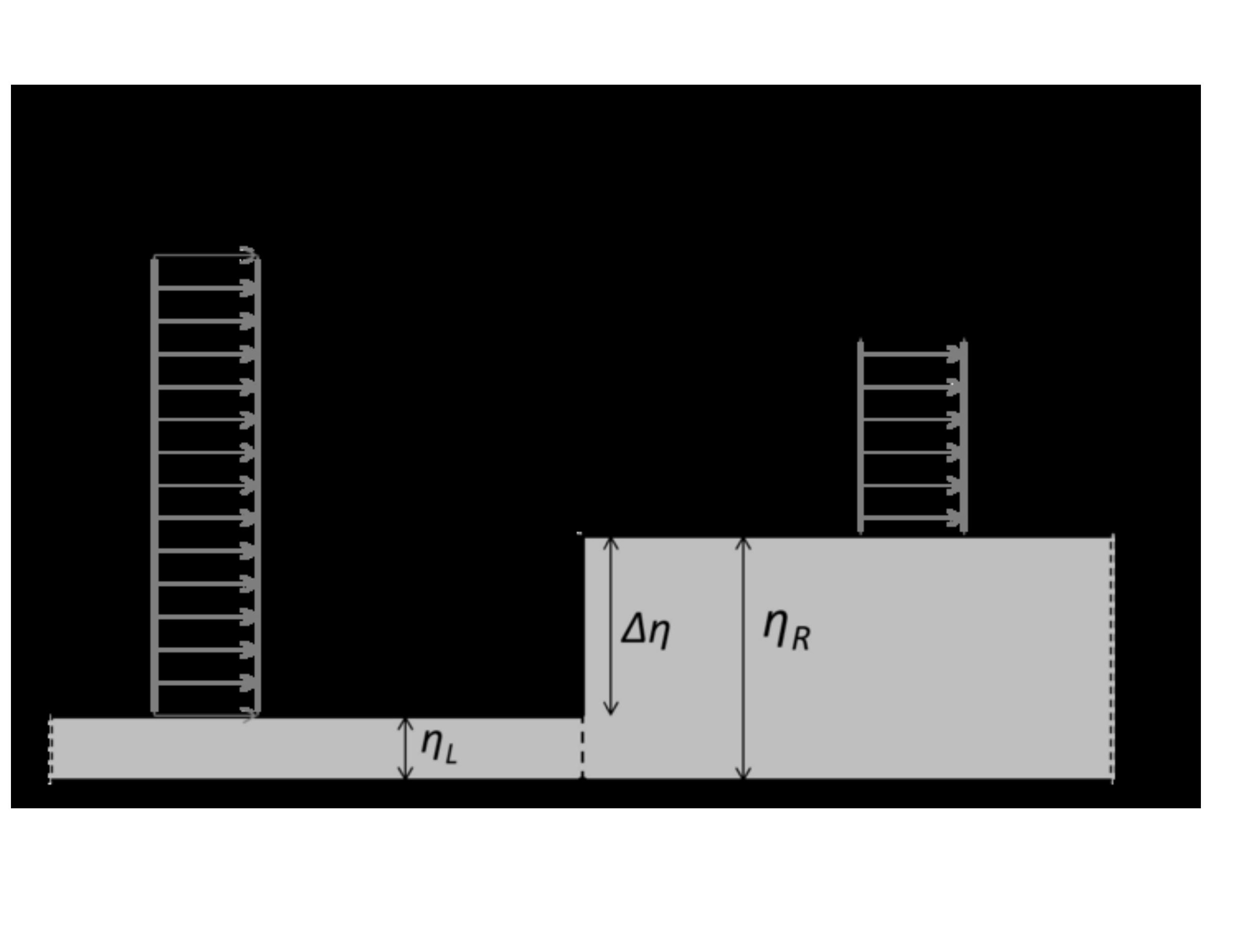


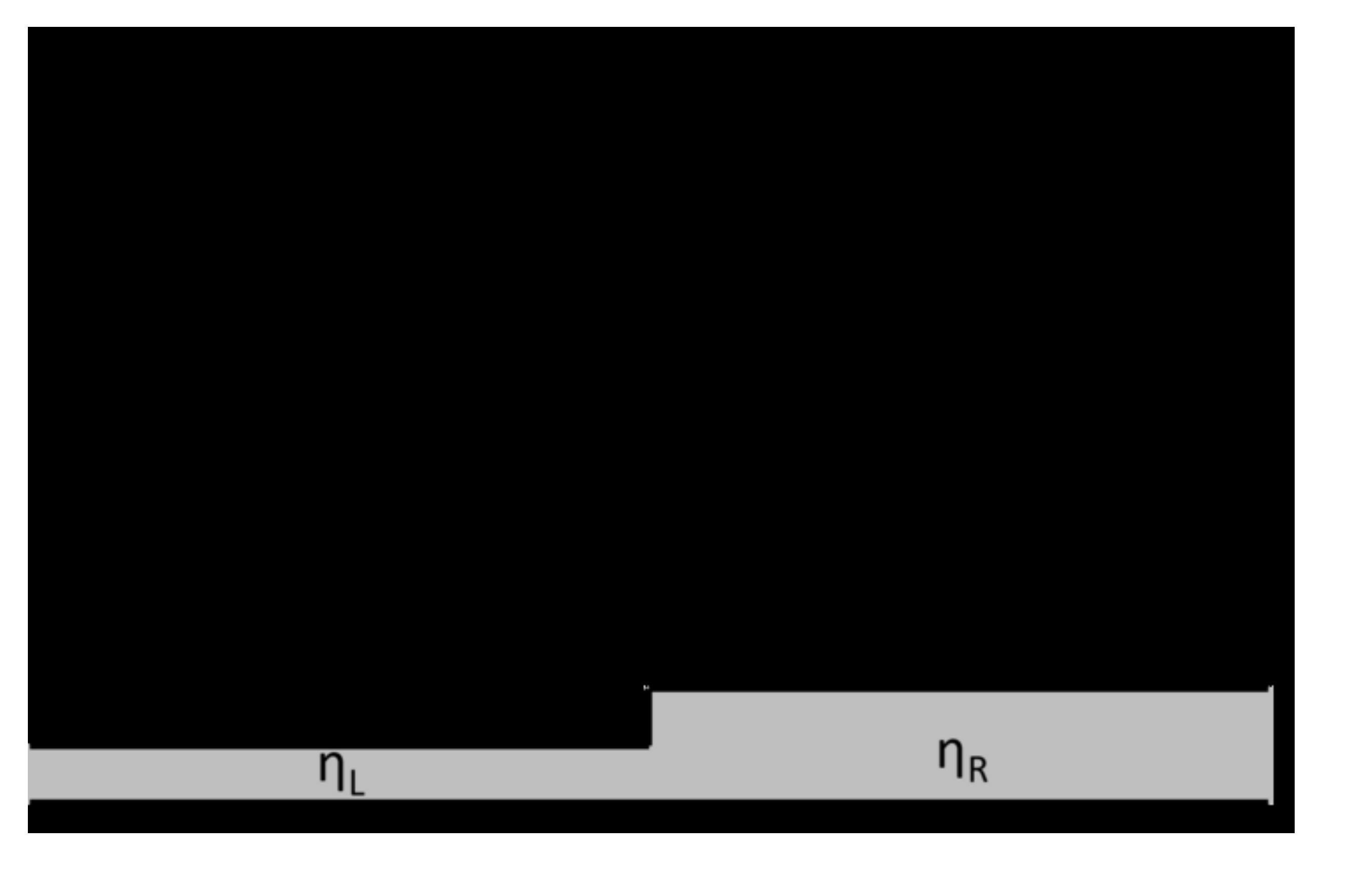

Figure

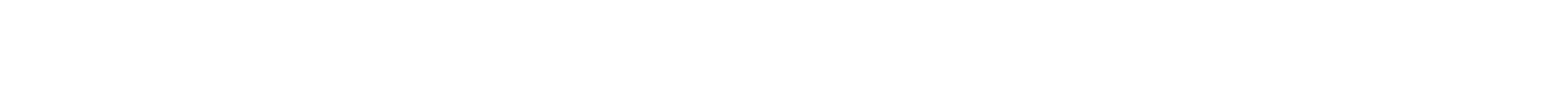

(n) 

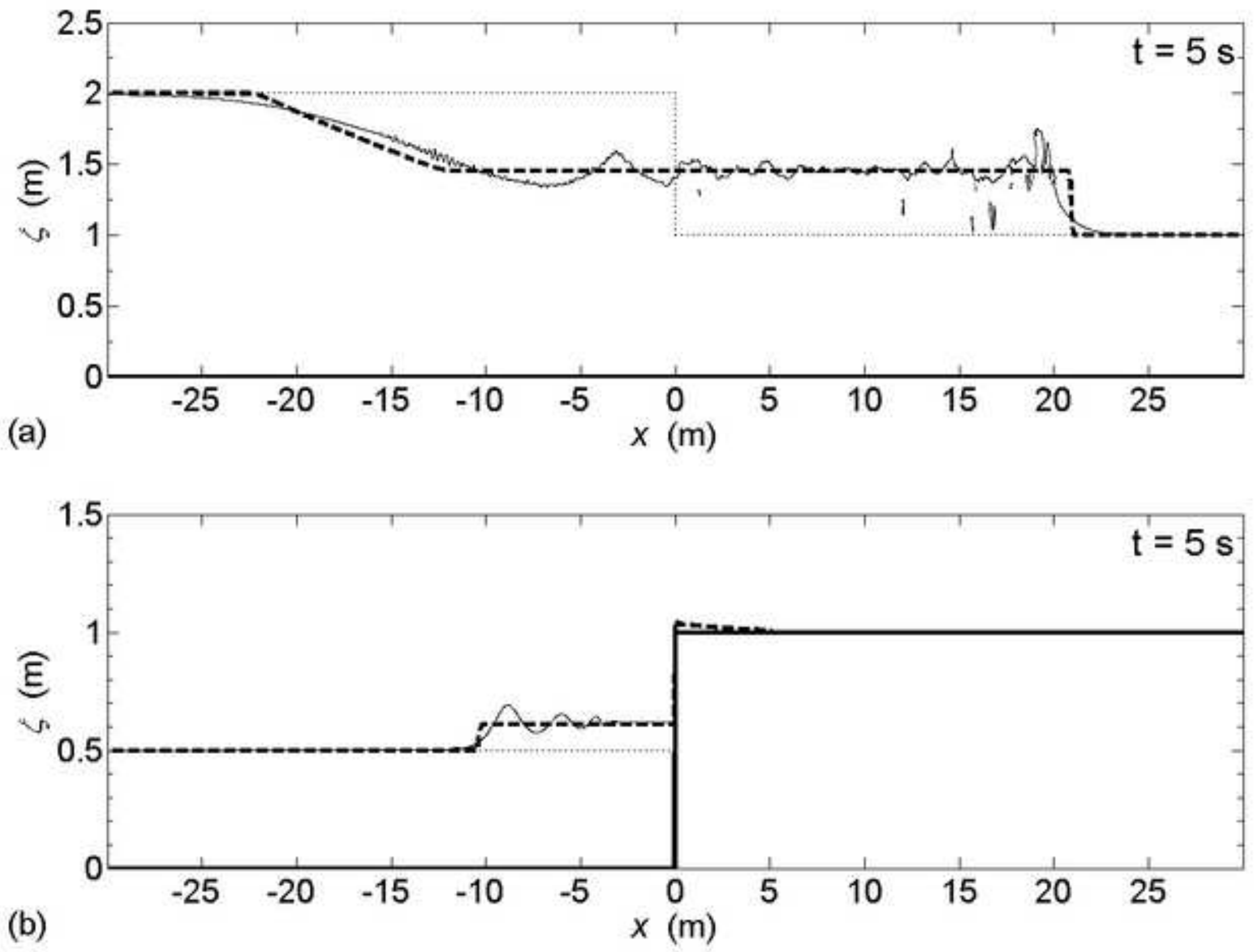

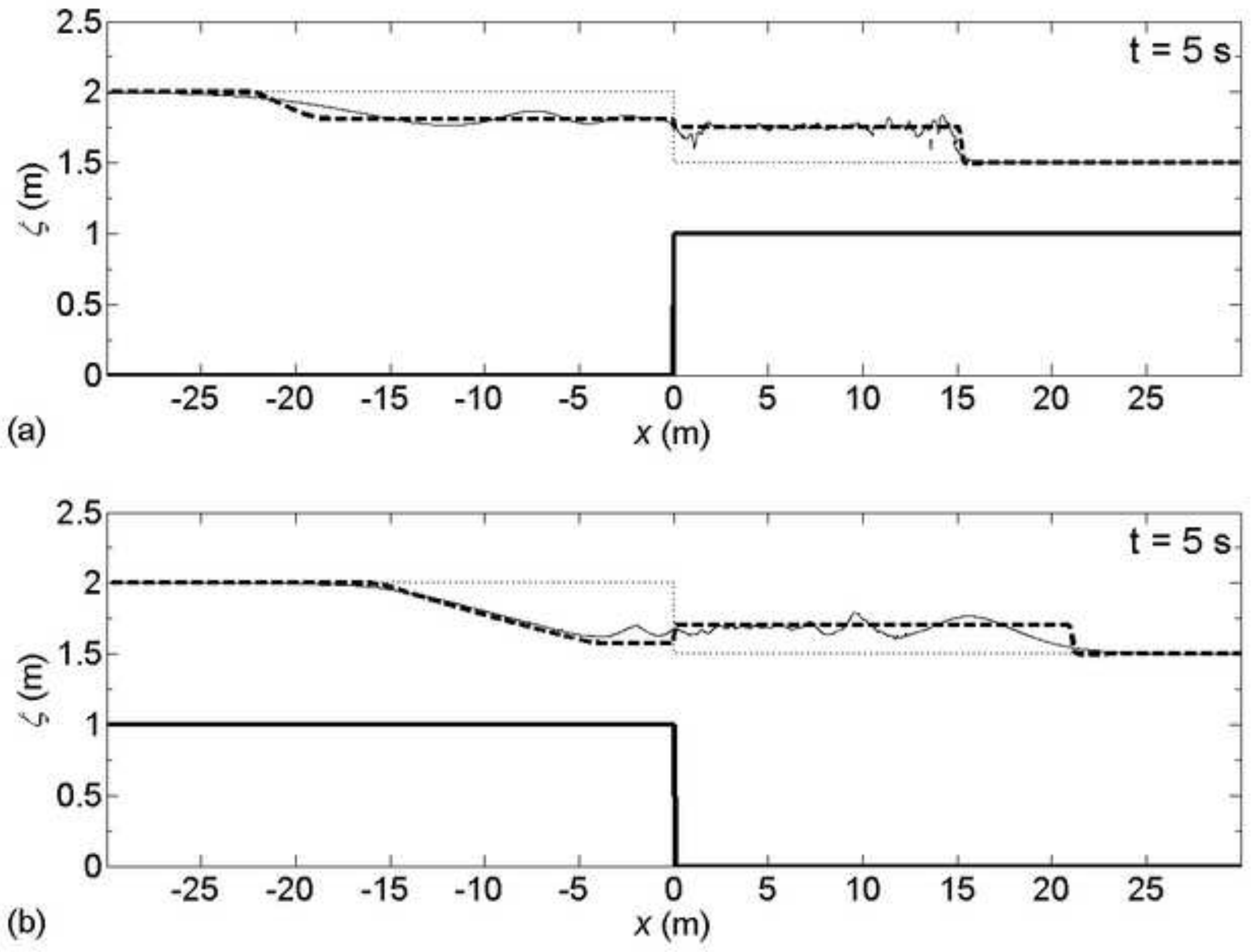

(a) 

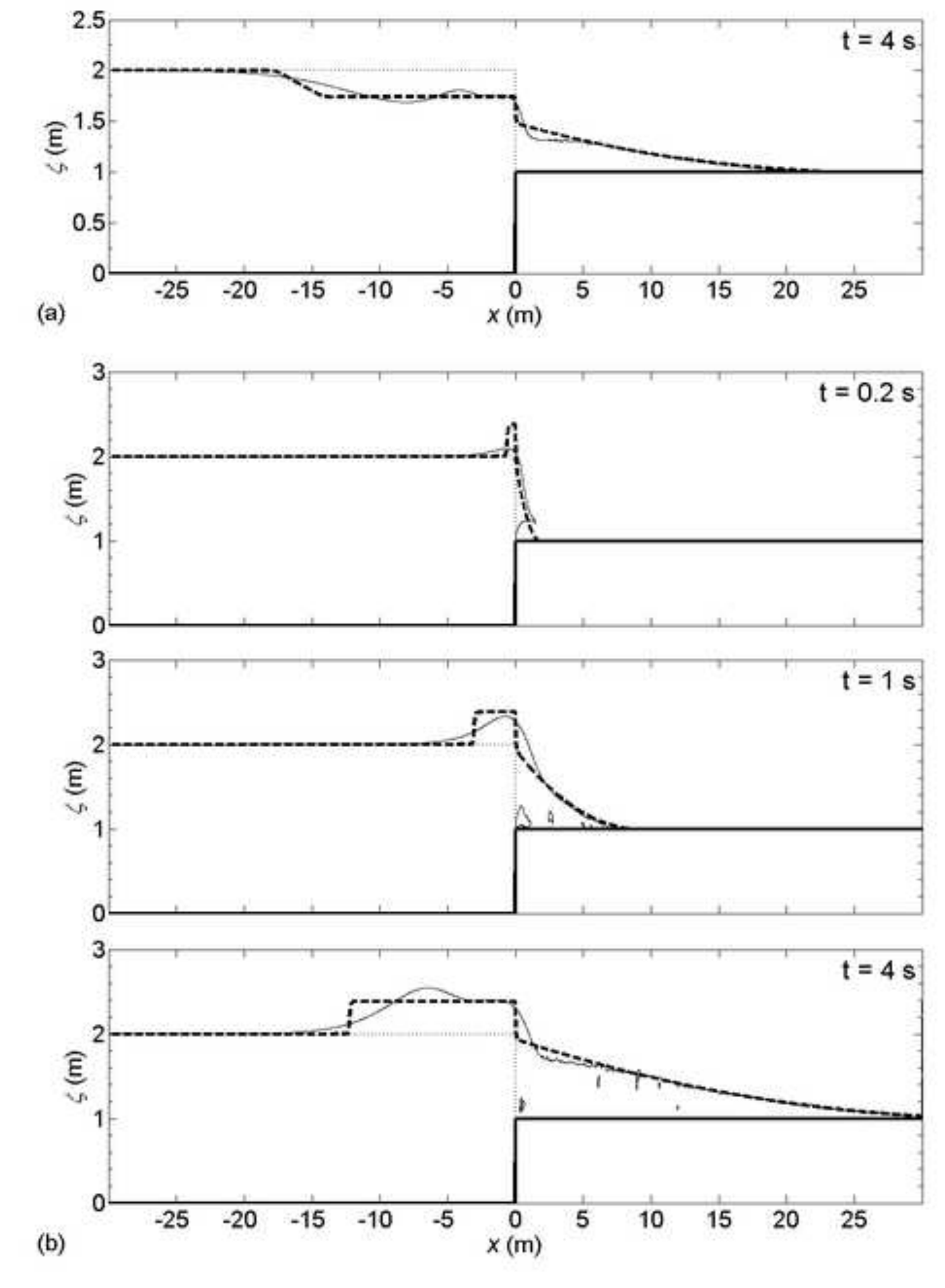

(b)

(a)

Figure 6 
Click here to download high resolution image
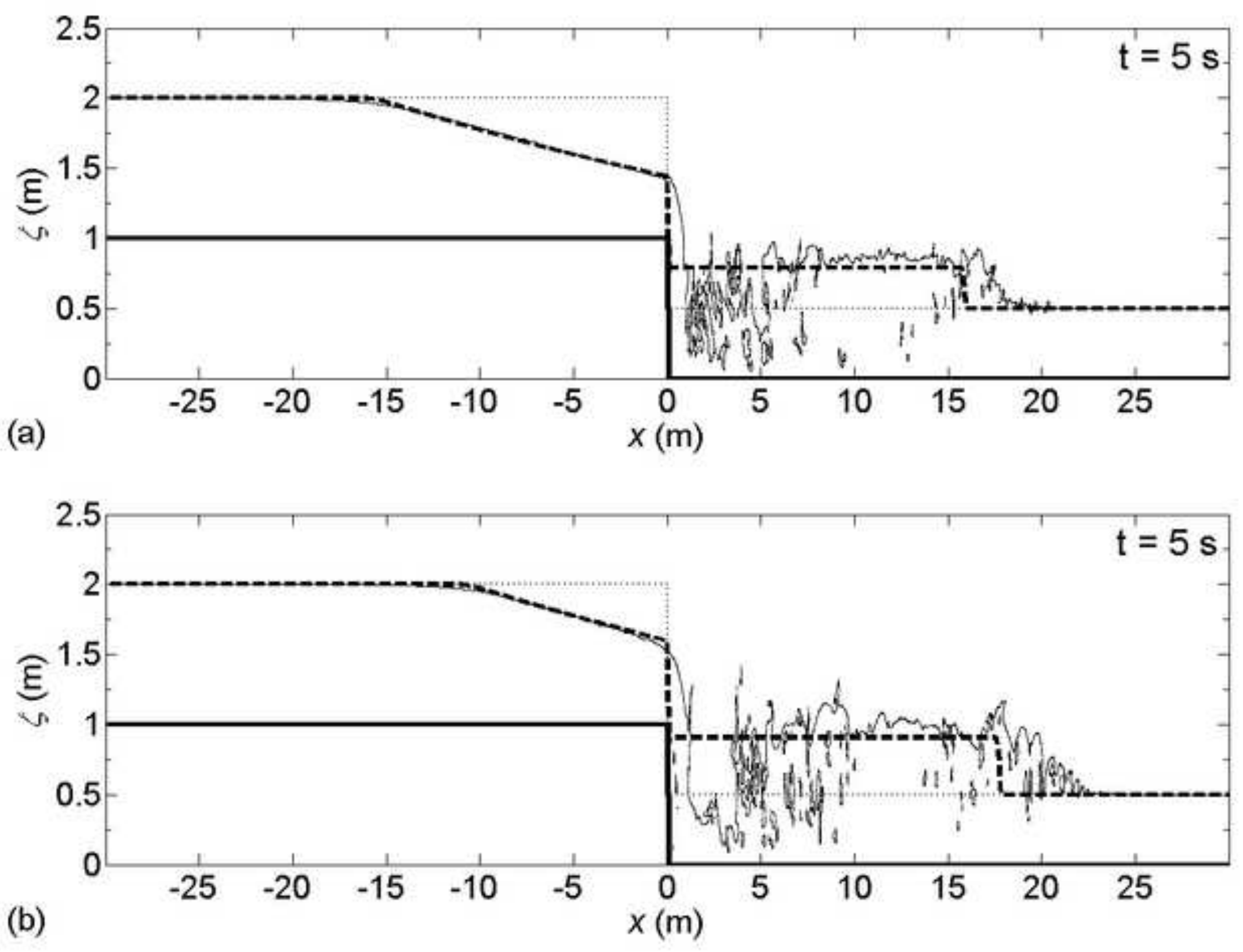

Click here to download high resolution image

(a) 

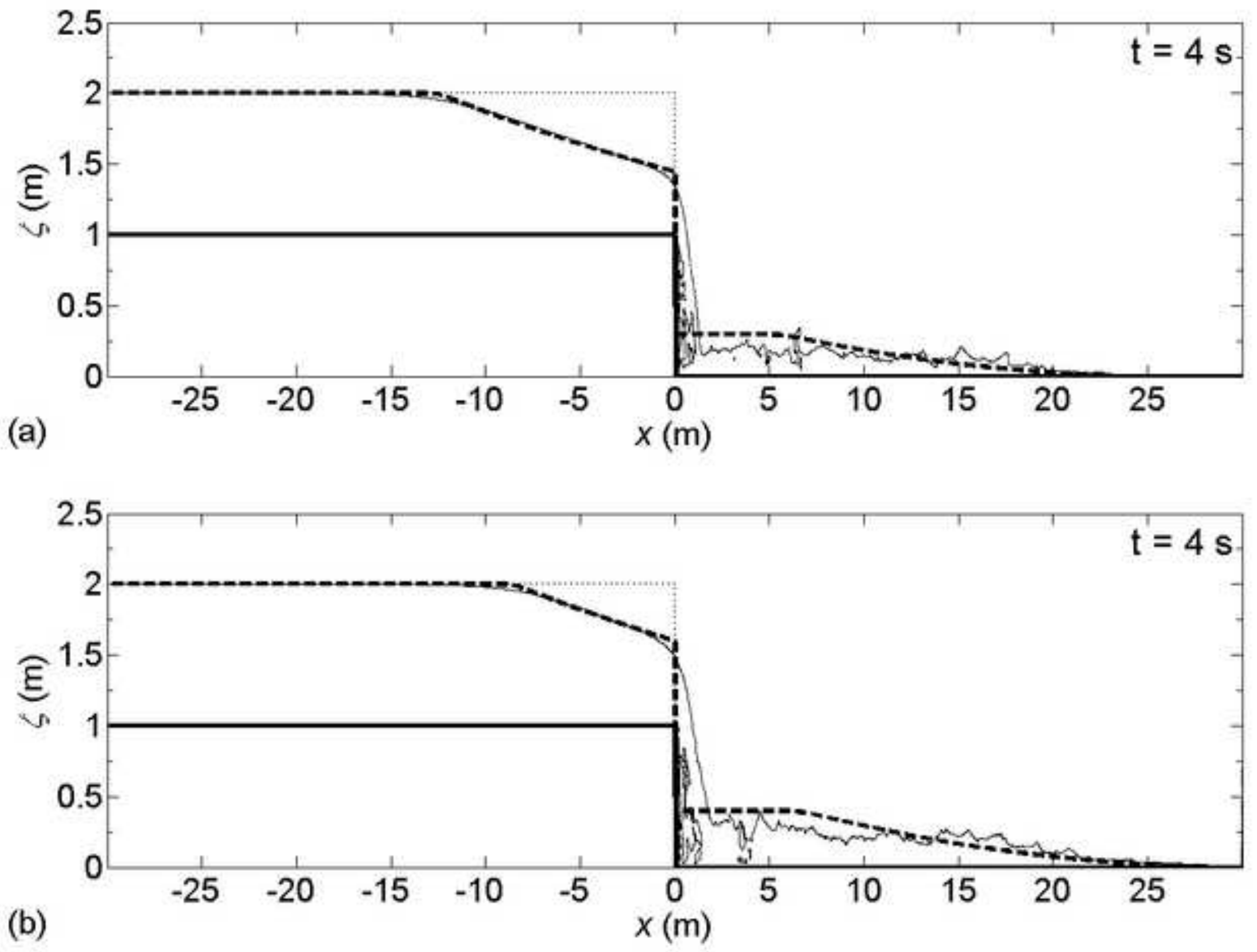

(b) $x(m)$

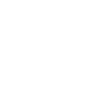




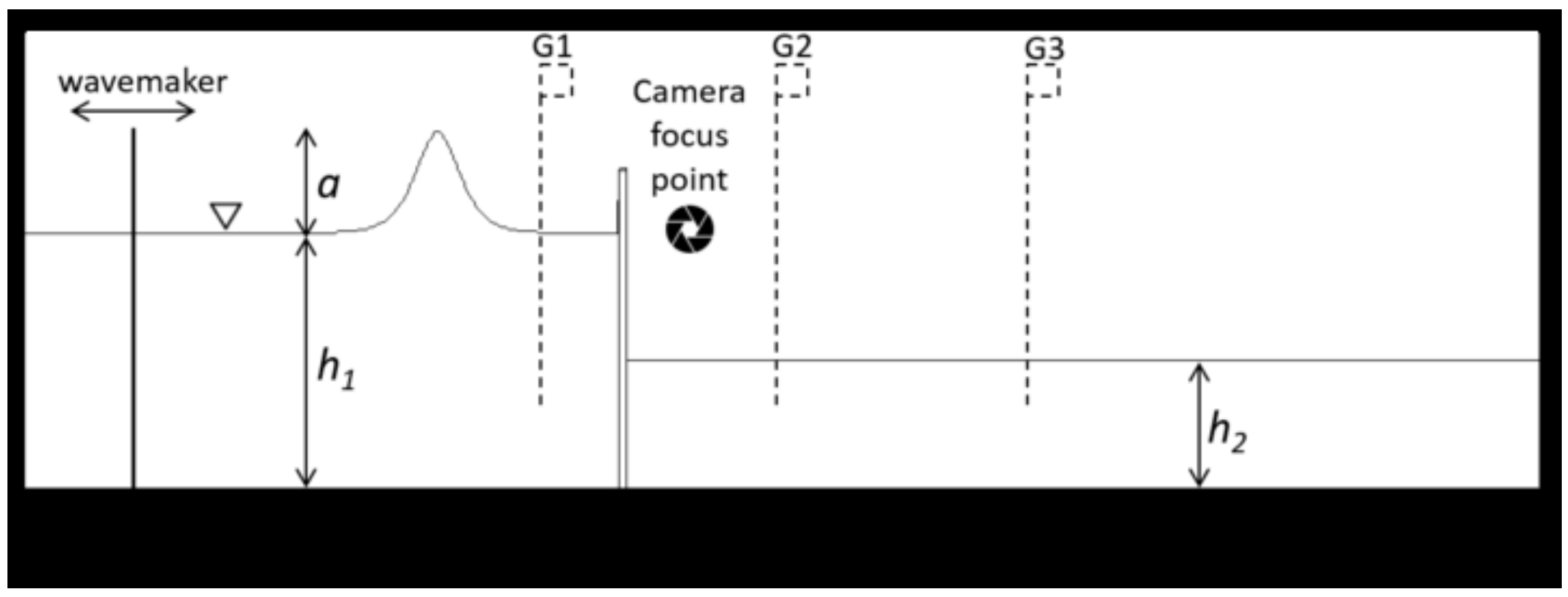



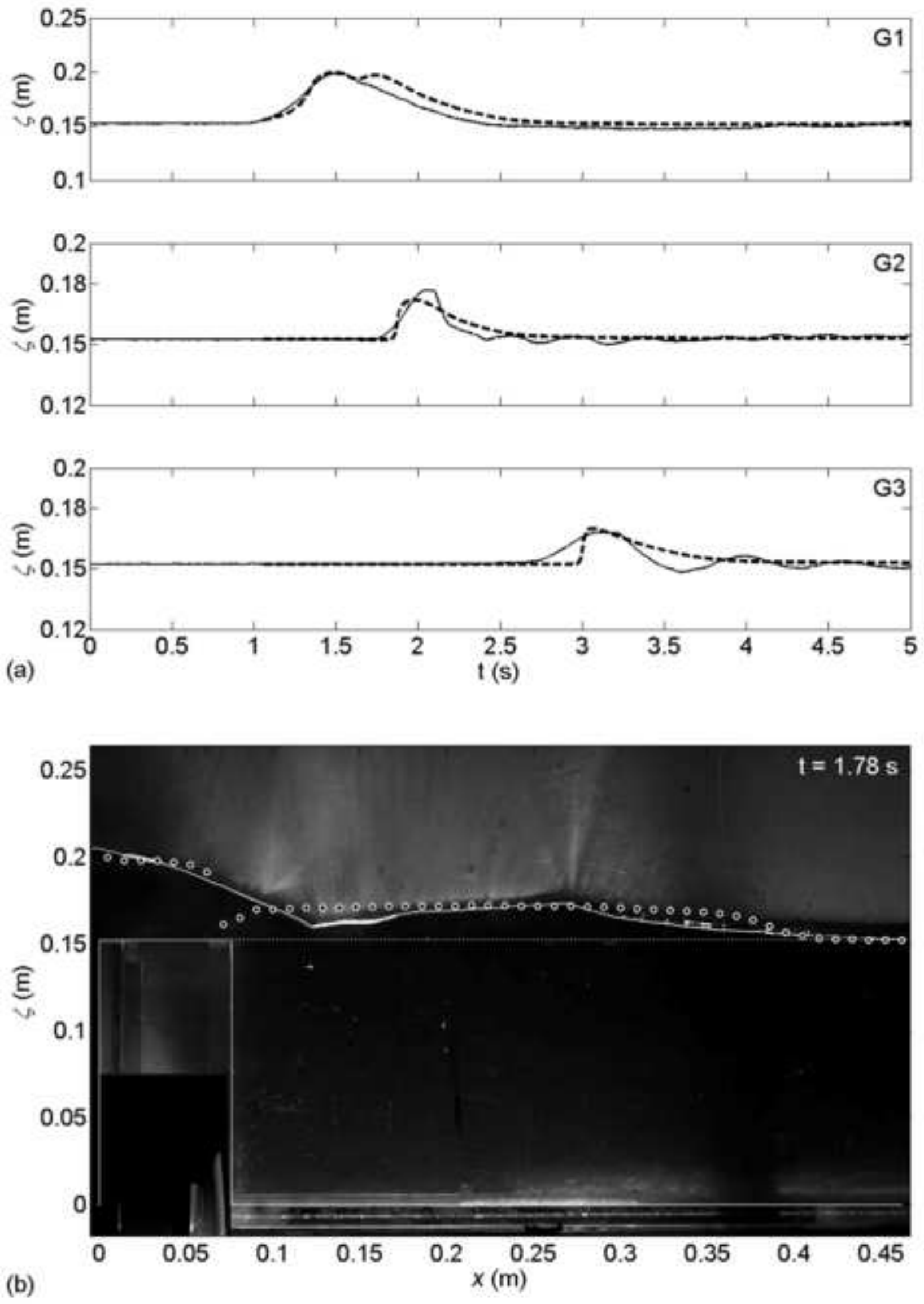

(b) 

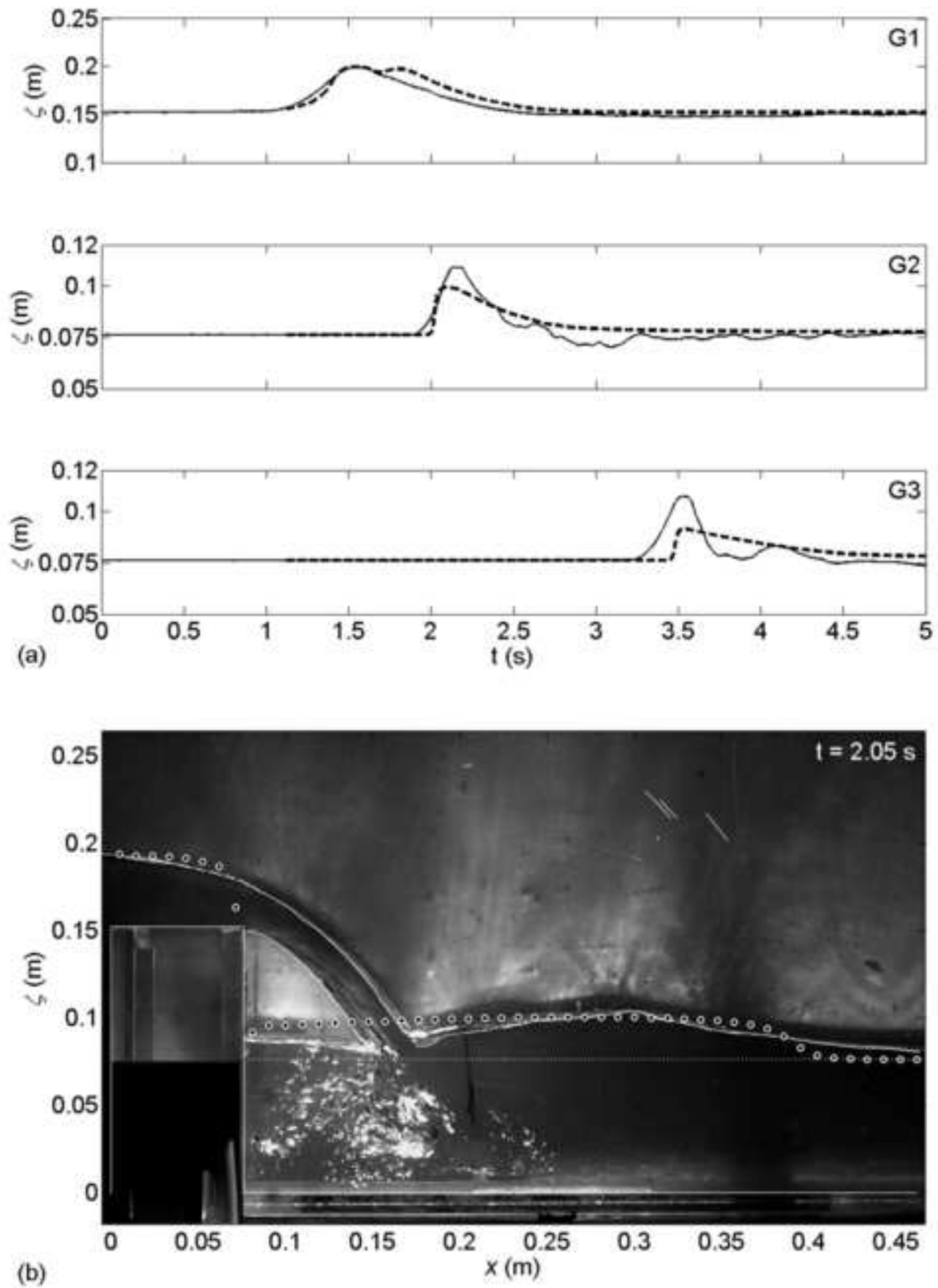

(b) 

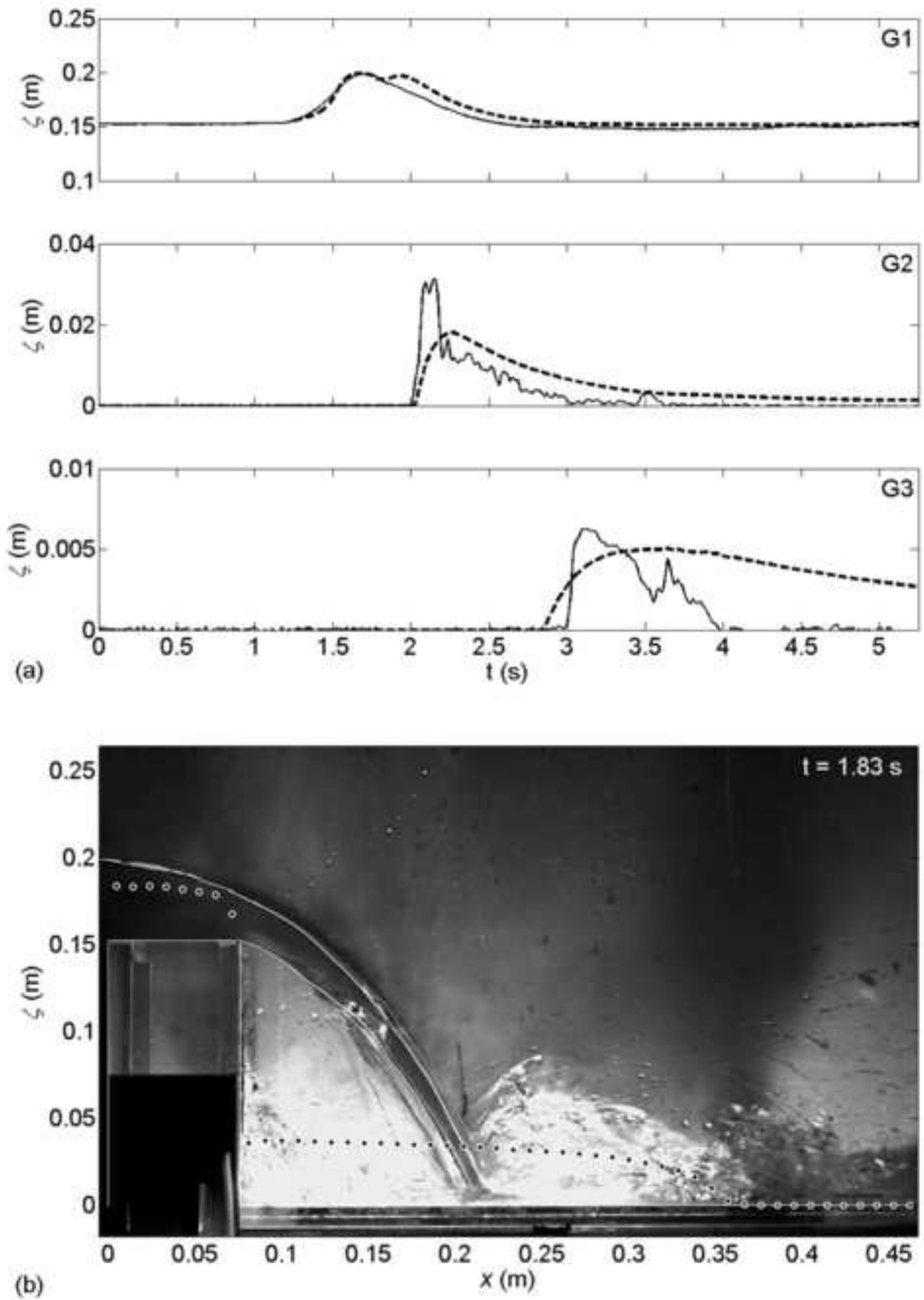

(b) 

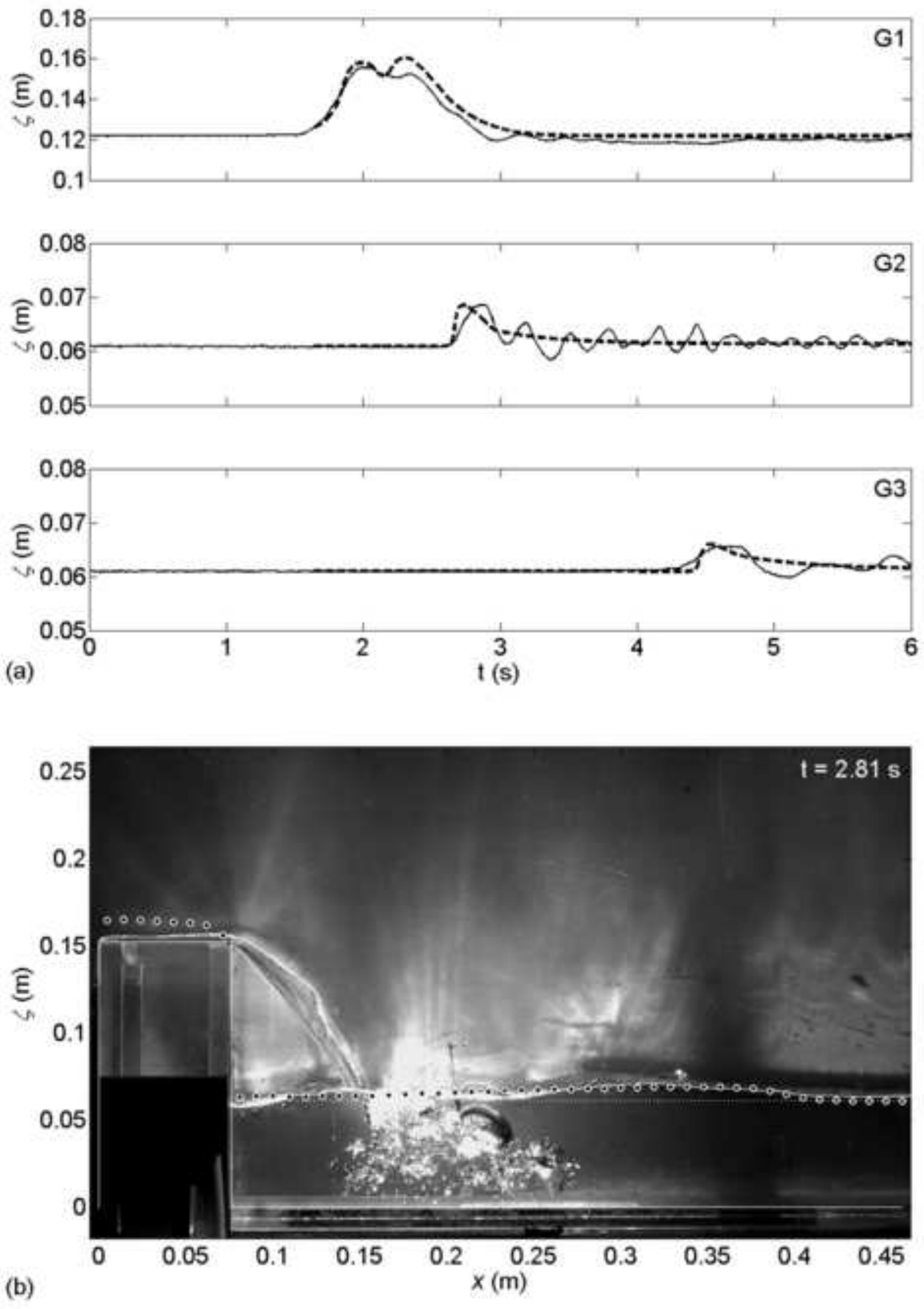

(b)

$$
\times(\mathrm{m})
$$

0.1

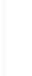

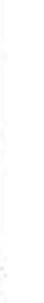

\section{Figure 13}

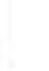

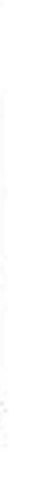



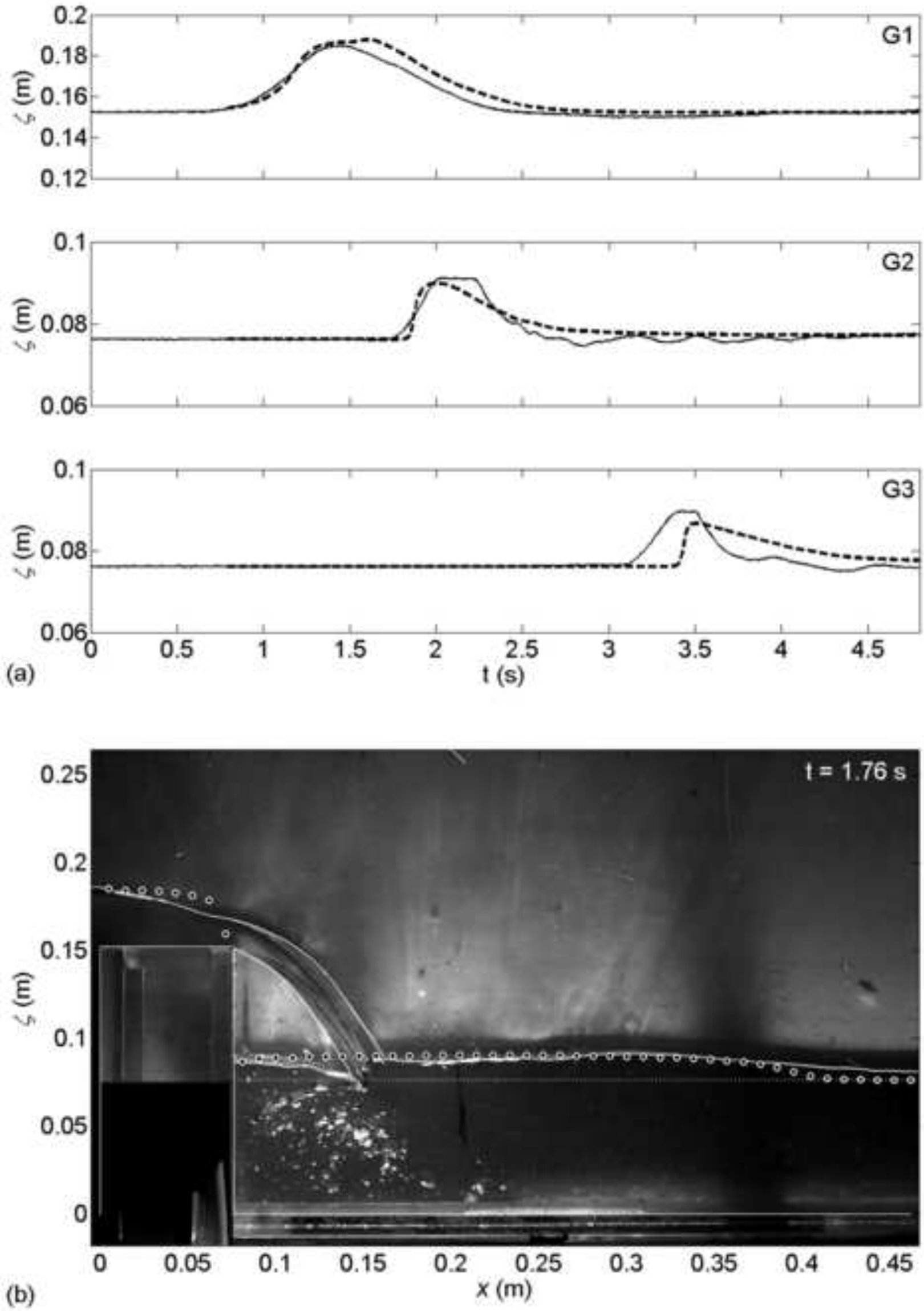

(b) 